\title{
Neuroprotective effects of Inter-alpha Inhibitor Proteins after hypoxic-ischemic brain injury in neonatal rats
}

\author{
Xiaodi Chen $\# 1,2$, Sakura Nakada ${ }^{\# 1,2}$, John E. Donahue ${ }^{2,3}$, Ray H. Chen ${ }^{1,2}$, Richard Tucker ${ }^{1}$, \\ Joseph Qiu ${ }^{4}$, Yow-Pin Lim ${ }^{2,4,5}$, Edward G. Stopa ${ }^{2,3}$, and Barbara S. Stonestreet ${ }^{1,2}$ \\ ${ }^{1}$ Department of Pediatrics, Women \& Infants Hospital of Rhode Island ${ }^{2}$ The Warren Alpert \\ Medical School of Brown University ${ }^{3}$ The Warren Alpert Medical School of Brown University, \\ Department of Pathology and Neurosurgery, Rhode Island Hospital ${ }^{4}$ ProThera Biologics, Inc., \\ Providence, RI ${ }^{5}$ The Warren Alpert Medical School of Brown University, Department of Pathology \\ and Laboratory Medicine, Providence, RI \\ \# These authors contributed equally to this work.
}

\section{Abstract}

Hypoxic-ischemic (HI) brain injury is one of the most common neurological problems occurring in the perinatal period. Hypothermia is the only approved intervention for neonatal HI encephalopathy. However, this treatment is only partially protective, has a narrow therapeutic time window after birth and only can be used to treat full-term infants. Consequently, additional therapies are critically needed. Inflammation is an important contributing factor to the evolution of HI brain injury in neonates. Inter-alpha Inhibitor Proteins (IAIPs) are immunomodulatory proteins with anti-inflammatory properties. We have previously shown that IAIPs reduce neuronal cell death and improve behavioral outcomes when given after carotid artery ligation, but before hypoxia in male neonatal rats. The objective of the current study was to investigate the neuroprotective effects of treatment with IAIPs given immediately or $6 \mathrm{~h}$ after $\mathrm{HI}$ in both male and female neonatal rats. HI was induced with the Rice-Vannucci method in postnatal (P) day 7 rats. After ligation of the right common carotid artery, P7 rats were exposed to 90 min of hypoxia (8\% oxygen). Human plasma-derived IAIPs or placebo (phosphate buffered saline) was given at zero, 24, and $48 \mathrm{~h}$ after HI. Brains were perfused, weighed and fixed $72 \mathrm{~h}$ after HI at P10. In a second, delayed treatment group, the same procedure was followed except that IAIPs or placebo were given at 6, 24 and $48 \mathrm{~h}$ after HI. Separate sham-operated, placebo-treated groups were exposed to identical protocols but were not exposed to carotid artery ligation and remained in room air. Rat sex was recorded. The effects of IAIPs on HI brain injury were examined using histopathological

Corresponding author: Barbara S. Stonestreet, M.D., The Warren Alpert Medical School of Brown University, Department of Pediatrics, Women \& Infants Hospital of Rhode Island, 101 Dudley Street, Providence, RI 02905-2499, Phone: (401) 274-1122 ext. 47429, Fax: (401) 453-7571, bstonestreet@wihri.org.

Publisher's Disclaimer: This is a PDF file of an unedited manuscript that has been accepted for publication. As a service to our customers we are providing this early version of the manuscript. The manuscript will undergo copyediting, typesetting, and review of the resulting proof before it is published in its final citable form. Please note that during the production process errors may be discovered which could affect the content, and all legal disclaimers that apply to the journal pertain.

Conflicts of interest

Y.-P. Lim and J. Qiu are employed by ProThera Biologies, Inc. All other authors declare no conflicts of interest. 
scoring and immunohistochemical analyses of the brain and by using infarct volume measurements on frozen tissue of the entire brain hemispheres ipsilateral and contralateral to $\mathrm{HI}$ injury. IAIPs given immediately after HI improved $(P<0.050)$ histopathological brain injury across and within the cingulate, caudate/putamen, thalamus, hippocampus and parietal cortex in males, but not in females. In contrast, IAIPs given immediately after HI reduced $(P<0.050)$ infarct volumes of the hemispheres ipsilateral to HI injury in similarly both the males and females. Treatment with IAIPs also resulted in higher $(P<0.050)$ brain weights compared with the placebo-treated HI group, reduced $(P<0.050)$ neuronal and non-neuronal cell death in the cortex and total hemisphere, and also increased the total area of oligodendrocytes determined by CNPase in the ipsilateral hemisphere and corpus callosum $(P<0.050)$ of male, but not female subjects exposed to HI. Delayed treatment with IAIPs $6 \mathrm{~h}$ after HI did not improve histopathological brain injury in males or females, but resulted in higher $(P<0.050)$ brain weights compared with the placebo-treated HI males. Therefore, treatment with IAIPs immediately after HI improved brain weights and reduced neuropathological brain injury and cell death in male rats, and reduced infarct volume in both male and female neonatal rats. We conclude that IAIPs exert neuroprotective effects after exposure to $\mathrm{HI}$ in neonatal rats and may exhibit some sex-related differential effects.

\section{Keywords}

Apoptosis; brain; Inter-alpha Inhibitor Protein; hypoxia-ischemia; neonate; neuroprotection; pathological score

\section{Introduction}

Neonatal hypoxic-ischemic (HI) brain injury is a leading cause of infant mortality and neurologic morbidities such as cerebral palsy, sensory and/or motor impairments, developmental delay, as well as cognitive abnormalities (Adhikari and Rao, 2017, Graham, et al., 2008). Hypothermia is the only currently available therapeutic intervention that can be used to treat HI encephalopathy in infants (Cotten and Shankaran, 2010, Klahr, et al., 2017, Osredkar, et al., 2014, Rumajogee, et al., 2016). However, there are limitations to the neuroprotective efficacy of this therapy, because it is only partially protective and has a narrow therapeutic time window of 6 hours after birth (Cotten and Shankaran, 2010, Klahr, et al., 2017, Osredkar, et al., 2014, Rumajogee, et al., 2016). Therefore, adjunctive treatment strategies are critically needed for neonates that have been exposed to HI.

Inter-alpha Inhibitor Proteins (IAIPs) are a family of structurally-related proteins found in high concentrations in human plasma, which have important immunomodulatory functions (Bost, et al., 1998, Businaro, et al., 1992, Fries and Blom, 2000, Kobayashi, 2006, Lim, 2013, Salier, et al., 1996, Zhu, et al., 2008). The major forms found in human plasma are Inter-alpha Inhibitor ( $I a I)$, consisting of two heavy chains and a single light chain, and Prealpha Inhibitor $(\mathrm{PaI})$, consisting of one heavy chain and one light chain (Potempa, et al., 1989, Zhu, et al., 2008). The light chain is also known as bikunin and contains two protease inhibitor domains resulting in its function as a serine protease inhibitor (Potempa, et al., 1989). The heavy chains play an important role in stabilizing and constructing extracellular matrix tissues, and synergize with the activity of bikunin (Zhu, et al., 2008). IAIPs inhibit 
destructive serine proteases, block pro-inflammatory cytokines, augment anti-inflammatory cytokine production, inhibit complement activation during systemic inflammation, and improve survival after sepsis in neonates and adults (Garantziotis, et al., 2007, Singh, et al., 2010, Yang, et al., 2002).

Previous work suggests that bikunin, also known as urinary trypsin inhibitor (UTI) or ulinastatin, has neuroprotective effects (Abe, et al., 1996, Feng, et al., 2014, Jiang, et al., 2016, Shu, et al., 2011, Yano, et al., 2003). Pretreatment with bikunin reduces the size of stroke-related infarcts in adult rats (Yano, et al., 2003), whereas treatment with bikunin reduces brain damage in young pigs after exposure to cardiopulmonary bypass (Wang, et al., 2013), reduces neuronal apoptosis in the hippocampus of adult gerbils after carotid artery occlusion (Abe, et al., 1996), and protects oligodendrocytes from apoptosis and increases remyelination in experimental autoimmune encephalitis in adult rats (Shu, et al., 2011). However, bikunin has a very short half-life (3-15 $\mathrm{min}$ ) because of its renal clearance (Sjoberg, et al., 1995) when compared with the blood-derived complexes of IAIPs, which have a 6- to 12-h half-life in rats (Wu, et al., 2004).

In recent studies, we have shown that treatment with blood-derived IAIPs decreases neuronal cell death, improves neuronal plasticity, attenuates complex auditory processing deficits and improves behavioral outcomes in male neonatal rodents after exposure to HI (Gaudet, et al., 2016, Threlkeld, et al., 2014, Threlkeld, et al., 2017). However, IAIPs in these studies were given after carotid artery ligation but before hypoxia (Gaudet, et al., 2016, Threlkeld, et al., 2014, Threlkeld, et al., 2017). Therefore, the administration of IAIPs in those studies represented a peri-insult treatment regimen, or a prevention strategy requiring prior knowledge of exposure to hypoxia (Gaudet, et al., 2016, Threlkeld, et al., 2014, Threlkeld, et al., 2017). Additionally, the studies examined only male rats, and did not examine overall histopathological brain injury, infarct volume, and white matter injury.

Based upon the above considerations, the objective of the current study was to investigate the potential neuroprotective effects of systemic treatment with IAIPs given immediately or $6 \mathrm{~h}$ after $\mathrm{HI}$ to both male and female neonatal rats. Changes in brain weight, histopathology, cell death, infarct volume, and oligodendrocyte area were examined in the neonatal rats treated with IAIPs immediately after exposure to HI. Changes in brain weight and histopathology were analysed in the delayed treatment group, which received IAIPs $6 \mathrm{~h}$ after HI. To study this we used the Rice-Vannucci method to induce HI in postnatal day (P) 7 neonatal rats. Although the rodent brain cannot be directly compared to the human neonatal brain, the brain of P7-10 rats is generally considered to be somewhat similar to the brain of near-term infants (Bockhorst, et al., 2008, Cowan, 1979, Dobbing and Sands, 1979, Semple, et al., 2013).

\section{Methods}

The experimental procedures in this study were performed after approval from the Institutional Animal Care and Use Committees (IACUC) of Brown University and of Women \& Infants Hospital of Rhode Island. All experimental procedures were carried out in 
accordance with the National Institutes of Health guide (NIH \#8023, 1978) for the care and use of laboratory animals.

\subsection{Animal preparation, surgical procedures, experimental design, and autopsies}

Pregnant Wistar rats were obtained from Charles River Laboratories (Wilmington, MA, USA) on embryonic day 15 or 16, and housed in a temperature-controlled, 12-h light/darkcycled facility with ad libitum access to food and water in the Animal Care Facility at Brown University. The dates upon which the rat pups delivered were confirmed and designated as P0. Litters were culled to a maximum of 10 pups on P1 such that there were as close to equal numbers of male and female pups as possible. On P7, the litters were randomly assigned to one of three groups: Sham-operated controls (Sham), HI placebo-treated (HIPL), or HI IAIP-treated (HI-IAIP). The sex of each subject was recorded.

The Rice-Vannucci method for the induction of HI was performed on P7 rats, which has been previously described in detail (Rice, et al., 1981). Briefly, the P7 rats were anesthetized with $4 \%$ isoflurane and maintained with $2 \%$ isoflurane during the surgical procedure. Body temperature was maintained at $36^{\circ} \mathrm{C}$ during surgery with a heating pad. The right common carotid artery was separated from the trachea and adjacent nerves, and double ligated with 5-0 silk sutures via a $0.5-1 \mathrm{~cm}$ vertical midline incision on the neck in the HI-PL and HIIAIP groups. In the Sham group, an equivalent incision was made on the neck, but the right common carotid artery was not ligated. The incision was closed, and each subject was marked with tail ink injections for identification (Neo-9, Animal Identification \& Marking Systems, Inc., Hornell, NY, USA) and then returned to the dam for recovery from surgery. The neonatal rats remained with the dams for $1.5-3 \mathrm{~h}$, after which the pups were placed in a hypoxia chamber (Biospherix, Parish, NY, USA) with $8 \%$ humidified oxygen and balanced nitrogen for 90 min (Fig. 1). The HI-PL and HI-IAIP subjects were monitored every $10 \mathrm{~min}$ during hypoxia, and body temperature was maintained at $36^{\circ} \mathrm{C}$ throughout the hypoxic exposure (Osredkar, et al., 2014). Sham subjects were placed in a similar container and remained in room air for $90 \mathrm{~min}$.

The experimental designs are illustrated in Fig. 1. After recovery from surgery and exposure to hypoxia, the immediate treatment group (Fig. 1A) was given three intraperitoneal (I.P.) injections of $30 \mathrm{mg} / \mathrm{kg}$ of human plasma-derived IAIPs (ProThera Biologies, Inc., Providence, Rl, USA) immediately (time zero), 24, and $48 \mathrm{~h}$ after the termination of hypoxia. In the delayed treatment group (Fig. 1B), the subjects were treated with I.P. injections of IAIPs $(30 \mathrm{mg} / \mathrm{kg}$ ) at 6,24 , and $48 \mathrm{~h}$ after the termination of hypoxia. In the Sham- and HI-PL-designated pups, I.P. injections of equivalent volumes of placebo (phosphate buffered saline, PBS) were administered as described above. Three separate time-matched, sham-treated groups were included for both the immediate and delayed treatment groups, as well as separately for the group on which infarct volumes were measured.

IAIPs were produced and purified as has been previously described (Lim, 2013, Opal, et al., 2011, Spasova, et al., 2014). Briefly, IAIPs were extracted from human plasma, purified with a monolithic anion-exchange chromatography (CIMmultus, BIA Separation, Villach, Austria) and eluted, and concentrated with a tangential flow filtration system (Labscale, 
Millipore) before undergoing purity analyses with SDS-PAGE, Western immunoblot, competitive IAIP ELISA and a standardized in vitro trypsin inhibition assay. The biological activity was measured based on the ability of IAIPs to inhibit the hydrolysis of the chromogenic substrate by trypsin (TAME or L-BAPNA). Inhibition was monitored by a decrease in the rate of change in absorbance/min at 405-410 $\mathrm{nm}$ wavelengths (Lim, et al., 2005).

The subjects were weighed before surgery and at the time of each IAIP or placebo injection. At $72 \mathrm{~h}$ after HI, the subjects were weighed, and sedated with ketamine ( $74 \mathrm{mg} / \mathrm{kg}$, I.P.) and xylazine (4 mg/kg, I.P.). Brain perfusion was performed with PBS and $4 \%$ paraformaldehyde (PFA) via cardiac puncture at a flow rate of $3 \mathrm{ml} / \mathrm{min}$. Thereafter, the brains were removed, weighed, post-fixed with PFA for $24 \mathrm{~h}$, and stored in $30 \%$ sucrose in phosphate buffer $(0.1 \mathrm{M})$ at $4^{\circ} \mathrm{C}$ before paraffin embedding for histopathological scoring and immunohistochemical staining (Threlkeld, et al., 2014) or before cryosectioning for the infarct volume analysis (Zhang, et al., 2004). The paraffin-embedded brain tissues were sectioned in coronal planes at $10 \mu \mathrm{m}$ thicknesses. One coronal section per brain containing the dorsal hippocampus (bregma $-3.36 \pm 1.4 \mathrm{~mm}$ ) was utilized to standardize the histopathological scoring and immunohistochemical analyses (Chen, et al., 2019, Paxinos and Watson, 2017). The brain sections for the infarct volume analysis were cryosectioned at a thickness of $20 \mu \mathrm{m}$.

\subsection{Histopathological scoring}

Paraffin-embedded brain sections from the immediate or delayed study groups (Fig. 1A and B) were stained with Luxol fast blue and hematoxylin-eosin (LFB \& FIE). Two boardcertified neuropathologists, who were not aware of study groups, scored the sections of the hemisphere ipsilateral to the FI I injury without knowledge of the group allocation using a modification of an established scoring system (Vannucci, et al., 2004). The histopathological scoring was used to examine neuronal and white matter injury as well as overall histopathological damage to the cingulate gyrus, parietal cortex, hippocampus, caudate/ putamen and thalamus brain regions semi-quantitatively. LFB staining was used to evaluate damage to white matter indicating myelin loss or degradation i.e decreased LFB staining or white matter injury. FIE staining was used to examine nuclear pyknosis, cytoplasmic reddening and condensation, and hyperchromatism that are indicative of neuronal injury and gliosis assessed by the presence of reactive gemistocytes, which are also indicative of damage to white matter (Brown and Brierley, 1972). FHI-related changes included parenchymal cystic necrosis and non-cystic necrosis and edema, myelin pallor, neuronal loss and reactive gliosis. The pathological criteria for FIE identification of reactive gliosis included the presence of reactive astrocytes, including increases in both the size and number of astrocytes, along with an overall increase in the cellularity of white matter. Reactive (gemistocytic) astrocytes are readily identifiable by HE staining as large astrocytes with plump, eosinophilic cytoplasm and eccentric nuclei.

Five brain regions were evaluated for evidence of HI-related injury including the cingulate gyrus, parietal cortex, hippocampus, caudate/putamen and thalamus. The scoring system of Vannucci et al. was modified as follows: a score of $0=$ no detectable HI damage; $1=$ focal 
loss of individual neurons; 2 = columnar damage involving multiple cortical neuronal layers, or in the hippocampus, caudate/putamen and thalamus as multiple or large foci of neuronal loss along with edema and reactive gliosis but without cystic infarction; and $3=$ severe cystic and non-cystic infarction, edema, myelin pallor and reactive gliosis (Vannucci, et al., 2004).

\subsection{Infarct volume measurement}

The neonatal rats used for the infarct volume measurements represented a separate group of animals from those used for the histopathological scoring and immunohistochemical analyses in the current study. Brains were divided into four or five $2 \mathrm{~mm}$ coronal sections using a brain sheer matrix (Zivic instruments, Pittsburgh, PA, USA), immersed in optimal cutting temperature (OCT) embedding medium, and frozen in a metal beaker filled with isopentane surrounded by crushed dry ice. Five cryosections $(20 \mu \mathrm{m})$ were obtained from each 2-mm section and mounted on gelatin-coated slides. We randomly selected every fourth cryosection to be stained with cresyl violet for evaluation of HI cell injury (Zhang, et al., 2004). The images of hemispheric and damaged areas were obtained using a Micropublisher 6 CCD Camera (Qimaging, Surrey, British Columbia, Canada) and analysed with ImageJ (NIH) without knowledge of the group assignment. The respective volumes were calculated from each measured area by multiplying the distance between sections. The infarct was calculated as a percentage of the ratio of the damaged volume to the volume of the total contralateral hemisphere with correction for hemispheric edema, according to the following formula: infarct $(\%)=[1-($ volume of total ipsilateral hemisphere - volume of infarct $) /$ volume of total contralateral hemisphere) $\times 100 \%$ (Sawada, et al., 2000, Swanson, et al., 1990).

\subsection{Immunohistochemical staining and quantification}

The paraffin-embedded brain sections were deparaffinized. Antigen retrieval was performed in a $120^{\circ} \mathrm{C}$ autoclave for $10 \mathrm{~min}$ with sodium citrate buffer $(10 \mathrm{mM}$ sodium citrate, $\mathrm{pH} 6.0)$ solution. The sections were blocked with a blocking solution (1\% bovine serum albumin, BSA, 5\% normal goat serum, NGS, TBST with $0.3 \mathrm{M}$ glycine) for $2 \mathrm{~h}$ at room temperature.

The neuronal and cell death immunohistochemical markers were determined as follows. Primary mouse anti-Fox3/neuronal nuclei (NeuN) monoclonal antibody (1:250; Abeam, Cambridge, MA, USA) was applied and incubated overnight at $4^{\circ} \mathrm{C}$, and Alexa Fluor ${ }^{\circledR} 594$ goat anti-mouse IgG secondary antibody (1:250; Invitrogen, Carlsbad, CA, USA) was then applied and incubated for $1 \mathrm{~h}$ at room temperature in the dark. An equilibration buffer (ApopTag® Plus Fluorescein In Situ Apoptosis Detection Kit S7111, EMD Millipore Corporation, Temecula, CA, USA) was applied and replaced with working strength Tdt enzyme (30\%, v:v). After incubation for $1 \mathrm{~h}$ at $37^{\circ} \mathrm{C}$, the Tdt enzyme reaction was terminated with working strength stop/wash buffer (3\%, v:v) for $10 \mathrm{~min}$ at room temperature. Working strength anti-digoxigenin conjugate was applied (47\%, v:v) and left to incubate for $30 \mathrm{~min}$ at room temperature.

After incubation with the secondary antibody, the slides containing the stained sections were dried and mounted with a medium containing 4', 6-diamidino-2-phenylindole (DAPI; Vector 
Laboratories, Inc., Burlingame, CA, USA). The NeuN- and ApopTag-stained sections were stored at $-20{ }^{\circ} \mathrm{C}$ until analysis.

DNA-DAPI complexes emit a bluish-white fluorescence when excited by ultraviolet light (wavelength of 385/30 nm). Nuclei with the blue DAPI fluorescence that co-localized with the Alexa Fluor 594 red fluorescence were considered NeuN-positive $\left(\mathrm{NeuN}^{+}\right)$cells. Similarly, nuclei with the blue DAPI fluorescence that co-localized with the green fluorescent cell death marker were considered ApopTag-positive (ApopTag ${ }^{+}$) cells (Figs. 7 and 8). ApopTag ${ }^{+} / \mathrm{NeuN}^{+}$cells denoted co-localization of the green fluorescence with the Alexa Fluor 594 red fluorescence. ApopTag ${ }^{+} / N_{\text {Non-Neuronal }}{ }^{+}$cells indicated green fluorescence without co-localization with the Alexa Fluor 594 red fluorescence.

ApopTag- and/or NeuN-labeled profiles within the cerebral cortex, hippocampus and total hemisphere ipsilateral to HI were imaged on a Zeiss Axio Imager M2 Imaging System microscope (Carl Zeiss, Inc., Jena, Germany) and quantified by the Stereo Investigator 10.0 fractionator probe software (MBF Bioscience, Williston, VT, USA) according to previously described methods (Threlkeld, et al., 2014). Sections of the cerebral cortex, hippocampus and total hemisphere were traced under 10x magnification. An automated stage paired with the Stereo Investigator was used to step systematically through the counting frames (Fractionator probe). ApopTag- and/or NeuN-labeled profiles were marked within each counting frame with a circular digital counting tool.

Oligodendrocytes were detected by identification of CNPase, a specific oligodendrocyte membrane enzyme. Primary mouse anti-CNPase (11-5B) monoclonal antibody (1:200; Abcam, Cambridge, MA, USA) was incubated overnight at $4^{\circ} \mathrm{C}$ after blocking for $2 \mathrm{~h}$. Thereafter, Alexa Fluor ${ }^{\circledR} 555$ goat anti-mouse IgG secondary antibody (1:500; Invitrogen, Carlsbad, CA, USA) was applied and incubated for $1 \mathrm{~h}$ at room temperature in the dark. The CNPase-stained sections were stored at $4{ }^{\circ} \mathrm{C}$ until analysis.

The CNPase marker was determined in the entire coronal section by whole brain imaging scanned on a Nikon Ti-E spinning disc confocal microscope using NIS Advanced Researcher software (Nikon Instruments, Inc., Melville, NY, USA). Positive CNPase staining areas were quantified in the corpus callosum, internal capsule and in the total hemisphere ipsilateral to the $\mathrm{HI}$ injury. Positive CNPase staining areas were determined by Fiji software as 8-bit grayscale images using a constant pixel threshold (Hagberg, et al., 2015, Lundgaard, et al., 2013, Mecha, et al., 2013, Schindelin, et al., 2012, Wang, et al., 2009). Results were expressed as a ratio of the positive CNPase staining area to the total hemisphere area ipsilateral to HI. All imaging and quantification were performed without knowledge of the study group assignments.

\subsection{Statistical analyses}

All results were expressed as mean \pm SEM. Body weight gain between treatment groups were compared across time points by two-factor analysis of variance (ANOVA) for repeated measures (Figs. 2A and 11A). One-way ANOVA was used to detect differences in brain weights and infarct volumes among the three treatment groups (Figs. 2B, 6 and 11B). If a significant difference was detected by ANOVA, the Fisher's least significant difference 
(LSD) method for multiple comparisons was used as a post-hoc test. SAS Proc GLIMMIX generalized mixed modeling (SAS Institute, Cary, NC, USA) was used for ordinal regression analyses of the histopathological injury scores for both the immediate (Fig. 5) and delayed (Fig. 12) treatment studies. Group differences were determined with least-squares means. If a significant difference was detected, the Tukey-Kramer test was used to detect specific differences after adjustment for multiple comparisons. The results from the ApopTag analysis (Fig. 8) were first $\log _{10}$-transformed to satisfy normality (Shapiro-Wilk normality test) and variance (Brown-Forsythe equal variance test) assumptions for ANOVA. Twofactor ANOVA for repeated measures was used to compare cell death across the cortex, hippocampus and total hemisphere among the treatment groups (Fig. 8). One-way ANOVA was also used to detect differences in the quantities of total ApopTag-positive (Total ApopTag ${ }^{+}$), ApopTag ${ }^{+} / \mathrm{NeuN}^{+}$, and ApopTag ${ }^{+} / \mathrm{Non}$-neuronal ${ }^{+}$cells between treatment groups. The Spearman's rank correlation compared the relationship between the immunohistochemical data and histopathological scores for the cerebral cortex ipsilateral to HI of male and female subjects (Fig. 9). One-way ANOVA was performed to compare the area of the CNPase expression within the corpus callosum, internal capsule and the positive area in the total hemisphere between the treatment groups (Fig. 10B). The Fisher's LSD post-hoc test was used for multiple comparisons if a significant difference was detected by ANOVA. $P<0.050$ was considered to indicate statistical significance.

\section{Results}

\subsection{Body weight gain and brain weights after immediate treatment with IAIPs or placebo in $\mathrm{HI}$ neonatal rats}

Fig. 2 contains the body weight gain (\%) plotted for the combined group of males and females, and separately for males and females. Body weight increased (ANOVA, $P<0.001$ ) in the Sham, HI-PL and HI-IAIP groups over the study periods (Fig. 2A; Male + Female, Male and Female). The increase in body weight over time was greater in the Sham than in the HI-PL (ANOVA, $P=0.003$ ) and HI-IAIP groups $(P=0.034$; Fig. 2 A; Male + Female). Likewise, the body weight increase over time was greater in the Sham than in the HI-PL group (Male; ANOVA, $P=0.006$ ) and greater in the HI-IAIP than in the HI-PL group in the males $(P=0.024)$. However, differences in body weight gain over time were not observed among the treatment groups in the female neonatal rats (Female; ANOVA, $P=0.252$ ).

Brain weights were higher in the Sham than in the HI-PL group (Fig. 2B; Male + Female; ANOVA, $P<0.001)$, and higher in the HI-IAIP than in the HI-PL group $(P=0.008)$. Brain weights were higher in the Sham than in the HI-PL group (Male; ANOVA, $P<0.001$ ), but were not significantly higher in the HI-IAIP than in the HI-PL group in the males (Male; ANOVA, $P=0.059)$. Brain weights were higher in the Sham than in the HI-PL group (Female; ANOVA, $P=0.009$ ) but did not differ between the HI-PL and HI-IAIP groups in the females (Female; ANOVA, $P=0.079$ ). 


\subsection{Systemic injections with IAIPs attenuate pathological brain injury in HI male neonatal rats}

Fig. 3 contains representative histopathological coronal brain sections from the Sham, HIPL, and HI-IAIP males (top row) and females (bottom row). The inset (Sham Male) shows the level in the brain at which the coronal sections were obtained. The section from the Sham control male brain shows no evidence of HI injury and represents a score of 0 . Both cerebral hemispheres are symmetrical with no cystic necrosis or non-cystic necrosis, edema, and no pallor of the myelin or loss of neurons. In contrast, the brain from the HI-PL male shows asymmetric hemispheres. There is extensive loss of brain parenchyma because of cystic necrosis and non-cystic necrosis with ventricular enlargement, pallor of the myelin, edema and hippocampal shrinkage and neuronal loss. There is widespread damage with involvement of adjacent parietal cortex, cingulate gyrus, hippocampus and deep gray structures (caudate/putamen and thalamus). This represents a score of 3. Inspection of the coronal section from the HI-IAIP male shows much less extensive damage than observed in the HI-PL male. The hippocampal structure is still relatively preserved in the HI-IAIP male rat section with less involvement of adjacent cerebral cortex and deep gray structures and reduced pallor of the myelin, gliosis and edema. This represents a score of 1 . The section from the Sham female also shows no evidence of $\mathrm{HI}$ injury and represents a score of 0 . The brain from the HI-PL female shows markedly asymmetric hemispheres with extensive loss of brain parenchyma, pallor of the myelin, edema and hippocampal shrinkage and neuronal loss. This represents a score of 3 . The coronal section from the HI-IAIP female shows similar damage to that of the HI-PL female. This represents a score of 3.

Fig. 4 contains representative higher magnification images of the histopathological findings in the cortex, hippocampus, caudate nucleus, cingulate and thalamus of Sham, HI-PL and HI-IAIP male rats. The section from the Sham control rat exhibits neuronal preservation with no evidence of underlying pathology in the five brain sections; this represents a score of 0 . The section from the HI-PL rat exhibits cystic and non-cystic necrosis, reactive gliosis, edema, pallor of the myelin and extensive neuronal loss in the cortex, hippocampus, caudate nucleus, thalamus and cingulate regions; these represent a score of 3. HI-related injury in the thalamus consisted of cystic and non-cystic infarction (see asterisk), neuronal loss, edema and reactive gliosis. Comparison of the sections from the HI-PL and HI-IAIP rats suggests that the HI-related injury in the five brain regions is reduced and less severe in the HI-IAIP male rat. In addition, there is relative absence of cystic and non-cystic necrosis and greater neuronal preservation in the section from the HI-IAIP male rat compared with the HI-PL neonatal rat. These represent a score of 1 . Sections from the female rats also indicate that the Sham control rat exhibits neuronal preservation with no evidence of underlying pathology in the five brain regions; this is a score of 0 . The section from the HI-PL female rat exhibits cystic and non-cystic necrosis, reactive gliosis, edema, pallor of the myelin and extensive neuronal loss in the cortex, hippocampus, caudate nucleus, and cingulate regions; these represent a score of 3. However, comparison of the sections from the HI-PL and HI-IAIP female rats do not suggest that the HI-related injury was attenuated to the same degree as in the male neonatal rats after treatment with IAIPs. 
The LFB\&HE-stained sections were evaluated independently by two board-certified neuropathologists without knowledge of the group designations. They utilized the 0-3 grade scoring system described above. The generalized linear model analysis demonstrated that treatment with IAIPs after HI reduced the average pathological scores across the evaluated brain regions in the HI-IAIP compared with the HI-PL group (Fig. 5A; Male + Female; GLIMMIX, $P=0.038$ ). The average pathological scores across the brain regions were also higher in the HI-PL and HI-IAIP compared with the Sham group (Fig. 5A; Male + Female; GLIMMIX, $P<0.001$ ). Post-hoc analysis identified significantly lower pathological scores in the HI-IAIP caudate/putamen and cingulate than in the respective brain regions in the HIPL group (Fig. 5A; Male + Female; $P=0.040$ and $P=0.002$, respectively). The average pathological scores across the brain regions were higher in the HI-PL and HI-IAIP males than in the Sham males (Fig. 5B; Male; GLIMMIX, $P<0.001$ ). The average pathological scores across the brain regions were lower in HI-IAIP males compared with HI-PL males (Fig. 5B; Male; GLIMMIX, $P=0.001$ ). Post-hoc analysis revealed that the pathological scores in each of the five brain regions examined were significantly lower in the HI-IAIP males than in the HI-PL males (Fig. 5B; Male; parietal cortex: $P=0.010$, hippocampus: $P=$ 0.026 , caudate/putamen: $P=0.001$, cingulate: $P<0.001$, thalamus: $P=0.010$ ). The average pathological scores across the brain regions did not differ (Fig. 5C; Female; $P=0.99$ ) between the HI-PL and HI-IAIP females, and the average pathological scores across the brain regions were higher in HI-PL and HI-IAIP females than in the Sham females (Fig. 5C; Female; GLIMMIX, $P<0.001)$. It is important to note that the histopathological injury in the cingulate of the HI-PL males was higher than in the HI-PL females (Fig. 5B and 5C; HIPL Males vs. Females for cingulate; GLIMMIX, $P<0.041$ ). However, the histopathological brain injury did not differ significantly in the other brain regions between the HI-PL male and female groups.

\subsection{Systemic injections with IAIPs decrease infarct volume of the hemisphere ipsilateral to $\mathrm{HI}$ injury in male and female $\mathrm{HI}$ neonatal rats}

Fig. 6A contains representative cresyl violet images of the coronal brain sections from the Sham, HI-PL and HI-IAIP males (top row) and females (bottom row). Compared to the HIPL group, reduced infarct areas were observed in the HI-IAIP group in both male and female animals. Compared to the Sham group, the percent infarct volumes were increased in the ipsilateral hemisphere (ANOVA, $P<0.001)$ of HI-PL groups in male + female (58.1 $\pm 2.5 \%)$, male $(58.5 \pm 1.9 \%)$, and female $(57.7 \pm 4.5 \%)$ neonatal rats (Fig. $6 \mathrm{~B})$. A reduction in the percent of infarct volume in HI-IAIP subjects was observed in male + female (34.7 $\pm 4.0 \%)$, male $(35.9 \pm 6 \%)$, and female $(33.6 \pm 5.6 \%)$ neonatal rats as compared to the HIPL subjects. Statistical differences were detected for percent infarct volumes between HI-PL and HI-IAIP subjects in male + female (ANOVA, $P<0.001)$, male $(P<0.001)$, and female $(P<0.001)$ neonatal rats, suggesting neuroprotection in the IAIP-treated HI-injured brain in neonatal rats.

\subsection{Systemic injections with IAIPs decrease neuronal and non-neuronal cell death in the cortex and total hemisphere of male $\mathrm{HI}$ neonatal rats}

Fig. 7 contains representative immunohistochemical images of the cerebral cortical sections from the Sham, HI-PL and HI-IAIP males (top row) and females (bottom row) of ApopTag- 
positive (green fluorescence), and neuronal, NeuN (Alexa Fluor 594 red fluorescence) profiles. The cell nucleus (blue fluorescent DAPI marker) was used as a counterstain. The inset on each image identifies the area of the brain from which the image was obtained. The white arrows indicate cells positive for the cell death marker (ApopTag). The white arrowheads indicate cells positive for both the NeuN and ApopTag markers. Co-staining was identified as NeuN-positive cells containing ApopTag reactivity in the nucleolus. The section from the cerebral cortex of the Sham male predominantly contains NeuN-positive cells with one ApopTag-positive cell (white arrow). The section from the HI-PL male contains numerous ApopTag-positive cells as well as ApopTag-positive and NeuN-positive cells (white arrowheads). The section from the HI-IAIP male contains fewer ApopTagpositive cells and some ApopTag-positive and NeuN-positive co-stained cells. The section from the Sham female is similar to that from the Sham male and the sections from the HI-PL and HI-IAIP females are similar to that from the HI-PL male.

In order to determine further the potential neuroprotective effects of IAIPs on brain injury after $\mathrm{HI}$ in neonatal rats, the number of ApopTag-positive cells ipsilateral to the $\mathrm{HI}$ hemisphere was quantified. Fig. 8 contains the average positive profiles of the total ApopTag ${ }^{+}, \mathrm{ApopTag}^{+} / \mathrm{NeuN}^{+}$, and ApopTag ${ }^{+} / \mathrm{Non}-\mathrm{Neuronal}^{+}$profiles in the cerebral cortex, hippocampus and total hemisphere of the Sham, HI-PL and HI-IAIP groups for the combined group of males and females, and separately for males and females. The total number of ApopTag ${ }^{+}$cells was higher (Male + Female; ANOVA, $P<0.001$ ) across the cortex, hippocampus and total hemisphere of the HI-PL than of the Sham group, but did not differ across the HI-PL and HI-IAIP groups (Male + Female; ANOVA, $P=0.059$ ). Post-hoc analysis revealed a higher amount of total ApopTag ${ }^{+}$cells (Male + Female; $P<0.001$ ) in the cortex, hippocampus and whole hemisphere of the HI-PL compared with the Sham group. Significant differences were detected in the number of ApopTag ${ }^{+} / \mathrm{NeuN}^{+}$cells in the cortex (Male + Female; ANOVA, $P=0.001)$ and whole hemisphere $(P=0.006)$ between the Sham, HI-PL and HI-IAIP groups. Post-hoc analysis showed that the amounts of ApopTag ${ }^{+} / \mathrm{NeuN}^{+}$ cells were higher in the HI-PL than in the Sham group in the cortex (Male + Female; $P<$ $0.001)$, and in the HI-PL than in the HI-IAIP $(P=0.034)$ and Sham $(P=0.002)$ groups in the whole hemisphere.

In the males, total ApopTag ${ }^{+}$cell numbers were higher across the cortex, hippocampus and total hemisphere in the HI-PL than in the Sham (Male; ANOVA, $P<0.001$ ) and HI-IAIP groups (Male; $P=0.005$ ). Post-hoc analysis revealed higher amounts of total $\mathrm{ApopTag}^{+}$cells in the cortex, hippocampus and total hemisphere of the HI-PL than of the Sham group (Male; $P<0.004)$, and lower amounts of total ApopTag ${ }^{+}$profiles in the cortex $(P=0.001)$ and total hemisphere $(P=0.010)$ of the HI-IAIP than of the HI-PL group. Significant differences were detected in ApopTag $/ \mathrm{NeuN}^{+}$cells in the cortex (Male; ANOVA, $P<$ 0.001 ) and whole hemisphere (Male; $P=0.006$ ) between the Sham, HI-PL and HI-IAIP groups. Post-hoc analysis indicated higher amounts of ApopTag ${ }^{+} / \mathrm{NeuN}^{+}$cells in the HI-PL than in the Sham (Male; cortex: $P<0.001$, hemisphere: $P=0.002$ ) and HI-IAIP groups in the cortex $(P=0.007)$ and whole hemisphere $(P=0.026)$. The numbers of ApopTag ${ }^{+} / \mathrm{Non}^{-}$ Neuronal ${ }^{+}$cells were higher across the cortex, hippocampus and total hemisphere in the HIPL than in the Sham group (Male; ANOVA, $P<0.001$ ). The numbers of ApopTag ${ }^{+} / \mathrm{Non}^{-}$ 
Neuronal $^{+}$cells were higher in the cortex (Male; $\left.P<0.001\right)$ and total hemisphere $(P=$ 0.009 ) in the HI-PL than in the HI-IAIP group.

In the females, total $\mathrm{ApopTag}^{+}$cell numbers were higher (Female; ANOVA, $P=0.001$ ) across the cortex, hippocampus and total hemisphere in the HI-PL than in the Sham group. Differences were not observed in the total number of ApopTag ${ }^{+}$cells across these regions between the HI-PL and HI-IAIP groups (Female; ANOVA, $P=0.820$ ). Differences also were not observed in the numbers of ApopTag ${ }^{+} / \mathrm{NeuN}^{+}$cells across the cortex, hippocampus and total hemisphere of the females among the study groups (Female; ANOVA, $P=0.500$ ). ApopTag $^{+} /$Non-Neuronal $^{+}$cell numbers were higher (Female; ANOVA, $P=0.001$ ) across the cortex, hippocampus and total hemisphere in the HI-PL than in the Sham groups, but did not differ between the HI-PL and HI-IAIP groups $(P=0.981)$. Therefore, IAIP treatment after exposure to $\mathrm{HI}$ appears to be associated with greater reductions in cell death in male compared with the female neonatal rats.

\subsection{Cerebral cortical ApopTag-labeled profile quantities correlate with cerebral cortical pathological scores in male and female $\mathrm{HI}$ neonatal rats}

Spearman rank correlations compared the ApopTag-labeled profiles in the cerebral cortex ipsilateral to $\mathrm{HI}$ injury with the pathological scores of the male and female subjects (Fig. 9). The total ApopTag ${ }^{+}$cells $(\mathrm{r}=0.842, \mathrm{n}=31, P<0.050), \mathrm{ApopTag}^{+} / \mathrm{NeuN}^{+}$cells $(\mathrm{r}=0.794, \mathrm{n}$ $=29, P<0.050)$, and ApopTag ${ }^{+} /$Non-Neuronal ${ }^{+}$cells $(\mathrm{r}=0.854, \mathrm{n}=31, P<0.050)$ in the male, and the total ApopTag ${ }^{+}$cells $(\mathrm{r}=0.806, \mathrm{n}=27, P<0.050)$, ApopTag $^{+} / \mathrm{NeuN}^{+}$cells $(\mathrm{r}$ $=0.664, \mathrm{n}=25, P<0.050)$, and ApopTag ${ }^{+} /$Non-Neuronal $^{+}$cells $(\mathrm{r}=0.795, \mathrm{n}=27, P<$ $0.050)$ in the female neonatal rats demonstrated positive linear correlations with their respective pathological scores.

\subsection{Systemic treatment with IAIPs increase the area stained by CNPase in the corpus callosum and total hemisphere ipsilateral to $\mathrm{HI}$ injury in male neonatal rats}

The oligodendrocyte membrane marker CNPase was utilized to examine the effects of IAIPs on injury to the white matter after HI because previous work has shown that the IAIP light chain (ulinastatin) increases the number of oligodendrocytes in an animal model of experimental autoimmune encephalomyelitis (Shu, et al., 2011). Fig. 10A contains representative immunohistochemical stitched, greyscale images of the coronal brain sections from the Sham, HI-PL and HI-IAIP males (top row) and females (bottom row) of areas stained for CNPase. A pixel threshold of 90-100 was set to determine positive CNPase staining. The section from the Sham male contains symmetrical cerebral hemispheres with clear evidence of CNPase staining in the corpus callosum (arrowhead) and internal capsule (arrow). The section from the HI-PL male contains asymmetrical hemispheres with minimal CNPase staining in the corpus callosum and internal capsule ipsilateral to the HI injury compared with the equivalent contralateral regions. The section from the HI-IAIP male contains relatively symmetrical CNPase staining in the callosum and internal capsule, and the CNPase-stained area of the ipsilateral HI hemisphere in the HI-IAIP male appears greater compared with that of the HI-PL male. The section from the Sham female was similar to that of the Sham male. The sections from the HI-PL and HI-IAIP females were similar to that of the HI-PL males. 
Fig. 10B contains the CNPase positively-stained areas as a ratio to the total $\mathrm{HI}$ ipsilateral areas for the corpus callosum, internal capsule, and total hemisphere. The CNPase positively-stained areas were greater (Male + Female; $P<0.001$ ) in the Sham compared with in the HI-PL group in the corpus callosum, internal capsule and in the total hemisphere. The CNPase-stained areas were greater in the corpus callosum, internal capsule and total hemispheric area of the Sham (Male; $P<0.002$ ) than in the HI-PL male group. Greater CNPase-stained areas were also detected in the corpus callosum (Male; $P=0.039$ ) and in the total hemisphere area $(P=0.003)$ of the HI-IAIP compared with the HI-PL male group. In contrast, CNPase-stained areas in the corpus callosum, internal capsule and total hemisphere were greater (Female; $P<0.040$ ) in the Sham than HI-PL female group but did not differ between the HI-PL and HI-IAIP female groups. Therefore, these findings can be interpreted to suggest that IAIP treatment after exposure to $\mathrm{HI}$ attenuates the reductions in oligodendrocyte areas in male but not in female neonatal rats.

\subsection{Body weight gain and brain weights after a 6-hour delay in treatment with IAIPs or placebo in $\mathrm{HI}$ neonatal rats}

Fig. 11 contains the body weight gain (\%) plotted for the combined group of males and females, and separately for the males and females that were treated with IAIPs or placebo 6 $\mathrm{h}$ after exposure to HI. Body weight increased (ANOVA, $P<0.050$ ) in the Sham, HI-PL and HI-IAIP groups over the study periods (Fig. 11A; Male + Female, Male and Female). The increase in body weight over time was greater (ANOVA, $P<0.001$ ) in the Sham than in the HI-PL and HI-IAIP groups (Fig. 11A; Male + Female). Likewise, the increase in body weight over time was greater in the Sham than in the HI-PL and HI-IAIP groups in the males (Male; ANOVA, $P<0.001$ ). The body weight gain in the females was greater (ANOVA, $P<0.001$ ) in the Sham group than in the HI-PL and HI-IAIP groups, but also greater in the HI-PL than in the HI-IAIP group $(P=0.012)$.

Brain weights were higher in the Sham than in the HI-PL and HI-IAIP groups (Fig. 11B; Male + Female; ANOVA, $P<0.002$ ). Brain weights were higher in the Sham (Male; ANOVA, $P<0.001)$ and HI-IAIP $(P=0.031)$ groups than in the HI-PL group in the males. Brain weights were likewise higher in the Sham than in the HI-PL and HI-IAIP groups in females (Female; ANOVA, $P<0.040$ ).

\subsection{Effects of systemic injections with IAIPs or placebo after a 6-hour delay on neuropathological scores in $\mathrm{HI}$ neonatal rats}

The LFB\&HE-stained sections for Fig. 12 were examined as described for Fig. 5 by two neuropathologists without knowledge of the group designations. Generalized linear model analysis demonstrated that treatment with IAIPs $6 \mathrm{~h}$ after HI did not significantly reduce the average pathological scores across the brain regions in the males + females, males or females (Fig. 12; GLIMMIX of averaged scores, males + females: $P=0.564$, males: $P=$ 0.087 , females: $P=0.403$ ). Furthermore, significant reductions in the individual pathological scores were not observed in the parietal cortex, hippocampus, caudate/putamen, cingulate, or thalamus of the HI-IAIP compared with HI-PL subjects for the males + females, males or females alone (GLIMMIX, $P>0.050$ ). However, it is important to emphasize that the pathological scores across the brain regions of the HI-PL males were higher (Fig. 5B vs. 
12B; GLIMMIX, average scores, $P=0.007$ ) in the group that received placebo immediately after HI compared with those that were treated with placebo $6 \mathrm{~h}$ after exposure to $\mathrm{HI}$ in spite of the fact that the HI exposures were identical in both groups. In addition, the pathological injury in the HI-PL group was higher in the cingulate (GLIMMIX, $P<0.001$ ) and thalamus $(P=0.013)$ of the males in the immediate placebo treatment group compared with the delayed placebo treatment group.

\section{Discussion}

The primary objective of the current study was to determine whether systemic treatment with the human blood-derived IAIPs attenuates overall neuropathological brain injury when administered after exposure to $\mathrm{HI}$ in neonatal rats. Although we have previously shown that treatment with IAIPs reduces neuronal cell death and improves behavioral outcomes in neonatal rats exposed to HI (Gaudet, et al., 2016, Threlkeld, et al., 2014, Threlkeld, et al., 2017), the IAIPs in our earlier studies were given as a peri-insult paradigm requiring prior knowledge of the hypoxic exposure. Moreover, only male neonatal rats were studied (Gaudet, et al., 2016, Threlkeld, et al., 2014, Threlkeld, et al., 2017). The major findings of the present study demonstrate that systemic treatment with the blood-derived IAIPs immediately after HI: 1) improves body weight gain over $72 \mathrm{~h}$ after exposure to $\mathrm{HI}$ in male neonatal rats; 2) attenuates HI-related reductions in brain weight; 3 ) improves overall neuropathological brain injury in male neonatal rats; 4) attenuates infarct volume in both male and female neonatal rats; and 5) reduces the HI-related increases in cell death and decreases in oligodendrocyte expression area in male neonatal rats. Some aspects of the beneficial neuroprotective effects were preferentially observed in the male neonatal rats. In addition, the effect of treatment $6 \mathrm{~h}$ after exposure to $\mathrm{HI}$ demonstrated that delayed treatment with IAIPs attenuated the HI-related decreases in brain weight in male rats, but did not improve body weight gain or significantly improve neuropathological injury after HI.

The neuropathological lesions caused by HI brain injury in the perinatal period include multifocal injury to the cerebral cortex, hippocampus and subcortical regions, which are most pronounced in the parasagittal cerebral cortex (Petersson, et al., 2002, Reddy, et al., 1998, Volpe, 1995). Additionally, cell death represents an important component of neurodegeneration after HI injury in the neonatal brain (Northington, et al., 2001, Northington, et al., 2001, Northington, et al., 2001, Northington, et al., 2005).

Inflammation is an important factor contributing to the development of brain damage after neonatal HI (Ferriero, 2004, McAdams and Juul, 2012). In premature infants, IAIP levels are decreased in association with systemic inflammatory disorders, including sepsis and necrotizing enterocolitis (Chaaban, et al., 2010, Chaaban, et al., 2009). In addition, both of these conditions are associated with increases in inflammatory markers and higher incidences of brain damage in premature infants (Shah, et al., 2008, Stoll, et al., 2004, Walsh, et al., 1989). These findings raise the interesting possibility that alterations in systemic and/or central nervous system IAIP levels could be important in HI-related brain damage in newborns. The results of the current study support this contention because systemic administration of blood-derived IAIPs attenuated the HI-related reductions in brain weight in the total group of males and females (Fig. 2B), and in the males, even when 
treatment was delayed for $6 \mathrm{~h}$ after HI (Fig. 9B). Brain weight has been previously shown to be a reasonable measurement of brain damage, because it correlates with morphometry after $\mathrm{HI}$ in neonatal rats, and is therefore a representative indicator of overall brain morphometric damage (Andine, et al., 1990). The present findings are also consistent with our previous report showing brain weight sparing when two doses of IAIPs were given after carotid artery ligation but before hypoxia (Threlkeld, et al., 2014). Consistent with the changes in brain weight, the HI-related histopathological changes were also attenuated by IAIPs in the male neonatal rats (Figs. 3-5) and brain infarct volumes were reduced in both male and female rats (Fig. 6).

In the current study, we used a scoring system adapted from Vannucci et al. (Vannucci, et al., 2004). Scoring of pathological brain sections by experienced neuropathologists (E.G.S. and J.E.D.) provides objective semi-quantitative examination of brain lesions, has been used extensively in studies of experimental and human brain injury (Hoque, et al., 2014), and has the advantage over determinants of specific cellular damage as it facilitates an overall assessment of brain damage by experienced examiners. Moreover, the positive correlations between the cell death marker and the histopathological scores (Fig. 9) support the validity of the histopathological scoring system. Results of the histopathological examinations confirmed that the blood-derived IAIPs were neuroprotective after systemic administration (Fig. 5). Although attenuation of injury was observed across the brain regions in the entire group of males and females (Fig. 5A), the beneficial neuroprotective effects were mainly observed in the male neonatal rats (Fig. 5B). The findings in the overall histopathological evaluation of brain injury are similar to our previous work showing that IAIPs reduced neuronal cell death as measured by Fluoro-Jade B staining of degenerating neurons (Threlkeld, et al., 2017). Consistent with our current and recent reports demonstrating neuroprotective effects after treatment with IAIPs (Gaudet, et al., 2016, Threlkeld, et al., 2014, Threlkeld, et al., 2017), bikunin exhibits neuroprotective properties in a variety of neurological conditions (Abe, et al., 1996, Feng, et al., 2014, Shu, et al., 2011, Yano, et al., 2003). In the adult, bikunin attenuates stroke-related brain injury by reducing brain volume loss and brain water content, down-regulating the expression of MMP-9 and reducing the loss of tight junction proteins, restoring blood-brain barrier function, and attenuating ischemia-related inflammatory responses (Chen, et al., 2014, Jiang, et al., 2016, Li, et al., 2017, Li, et al., 2017, Liu, et al., 2017). Bikunin has also been shown to reduce jugular venous superoxide radical production, oxidative stress, inflammation, and endothelial activation after forebrain ischemia caused by bilateral carotid artery occlusion and reperfusion after hemorrhagic hypotension (Koga, et al., 2010). Whether similar factors contribute to the neuroprotection observed after treatment of HI with IAIPs in the neonate cannot be determined from our current study and require future investigation. Although the current studies did not address the potential long-term effects of treatment with IAIPs on brain injury in neonatal rats, the effects appear to be durable based upon our previous report showing prolonged beneficial neuropathological and behavioral outcomes in adult rats after IAIP treatment at birth (Gaudet, et al., 2016, Threlkeld, et al., 2014).

Although the scoring of pathological brain sections by experienced neuropathologists has advantages over specific cellular determinants of brain injury because it facilitates an overall assessment of total and regional brain damage (Figs. 3, 4, 5), the histopathological scoring in 
our study was performed upon a single coronal brain section. In this regard, measurements of the percent of infarct volume (Fig. 6) that account for the injury across the entire brain is more likely to exhibit greater sensitivity, accuracy and to be more comprehensive than the semi-quantitative scoring methodology. In contrast to the histopathological scoring, cell death findings and CNPase immunohistochemical detection measures, which identified IAIP-related beneficial effects in the male, but not in the female neonatal rats, the infarct volume measures detected significant neuroprotective efficacy both male and in female neonatal rats after exposure to HI (Fig. 6). Therefore, immediate treatment with IAIPs exerts significant neuroprotective effects in both male and female neonatal rats based upon the findings of the reduced infarct volumes. Several possibilities could account for the discrepancies in our findings using the different methodologies. As described above, the use of scoring and immunohistochemical methodologies were based a single randomly selected coronal section of the brain that contained the dorsal hippocampus (bregma $-3.36 \pm 1.4 \mathrm{~mm}$ ) to standardize both histopathological and immunohistochemical analyses. Therefore, the greater sensitivity of the infarct volume measure could account for our ability to detect a protective effect of treatment with IAIPs in the group of female neonatal rats. Consequently, based upon the discrepancies in our findings, multiple different levels of coronal brain sections would need to be investigated to evaluate additional measures of pathological scoring, neuroinflammation, cell death, myelination, and astrogliosis analyses in future studies.

Although the HI-related decreases in brain weight were attenuated after delayed treatment with IAIPs in the males, the histopathological damage was not significantly decreased (Fig. 12). In this regard, it is important to note that the histopathological injury was significantly more severe in the group of neonatal male rats receiving placebo treatment immediately after $\mathrm{HI}$ compared with those receiving placebo $6 \mathrm{~h}$ after HI (Figs. 5B and12B). The reason for the unanticipated differences cannot be discerned from our studies, however, since the HI treatment and environmental temperatures were identical in both groups, it remains plausible that the neuroprotective effects of the IAIPs were not detected in the delayed group, because the injury was not sufficiently severe to detect differences between the IAIP and placebo groups (Fig. 12B). In addition, inadvertent variations among different litters could have contributed to the greater severity of injury in the group treated with placebo immediately after HI compared with $6 \mathrm{~h}$ after HI (Oakden, et al., 2002, Rumajogee, et al., 2016). The 6-h delay in treatment with IAIPs was based upon the recommended time window after birth in which therapeutic hypothermia is considered effective (Gunn, et al., 1997, Shankaran, et al., 2005). Nonetheless, it is also important to consider that the lifespan of rodents and humans differ greatly such that the equivalent of $6 \mathrm{~h}$ for the human newborn is most likely much shorter for a rodent (Andersen, 2003, Boxenbaum, 1982).

The findings of our study on cell death (Figs. 7 and 8) suggest that increases in cell death were observed $72 \mathrm{~h}$ after exposure to HI consistent with previous reports (Northington, et al., 2011, Northington, et al., 2001, Northington, et al., 2001, Northington, et al., 2001, Northington, et al., 2005). Increases in cell death were observed in both sexes, but increases in neuronal (NeuN-positive cells) cell death were more apparent in male than in female neonatal rats (Fig. 8). Although the increases in non-neuronal cell death were observed in both sexes, treatment with three doses of IAIPs attenuated the increases in HI-related cell 
death in both neuronal and non-neuronal cells in male, but not in female rats. The effects of IAIP treatment on cell death were generally similar to the results shown in the neuropathological scores (Fig. 5). Consistent with the reductions in cell death observed after systemic IAIP treatment in neonatal rats exposed to HI, treatment with bikunin inhibited ischemia-induced apoptosis in the hippocampus of adult gerbils and reduced neuronal apoptosis $72 \mathrm{~h}$ after cardiac arrest and return of spontaneous circulation in the cortex of adult male rats (Cho, et al., 2015, $\mathrm{Hu}$, et al., 2013).

Oligodendrocyte function is important for myelination and maintenance of homeostasis in the CNS, supporting axonal integrity and signaling with other cell types (Ligon, et al., 2006, Shindo, et al., 2016). White matter injury with loss of myelinating oligodendrocytes is observed after perinatal HI brain injury (Martinez-Biarge, et al., 2012). The HI-related decreases in oligodendrocyte area (Fig. 10B) are consistent with previous work (Fan, et al., 2005, Qiao, et al., 2018, Shindo, et al., 2016). Immediate treatment with IAIPs improved HIinduced white matter damage by increasing the quantity of CNPase-stained areas in the corpus callosum and total ipsilateral hemisphere in HI-injured males, but not in the females (Fig. 10B). Consistent with our findings, treatment with bikunin attenuates apoptosis of oligodendrocytes and increases re-myelination in experimental autoimmune encephalitis in adult rats (Shu, et al., 2011). Our IAIP-related decreases in white matter injury are consistent with decreases in cell death (Fig. 8) and improved pathologic scores (Fig. 5) in the male, but not in female neonatal rats. Nonetheless, it is important emphasize that these measures were performed on a single coronal brain section at the level of the dorsal hippocampus.

Therefore, it is not surprising that the IAIP-related improvements were similar for these three measures of injury.

The study subjects in each group gained body weight during the 72-h study period as expected (Figs. 2 and 11). Although the placebo-treated group also gained weight after exposure to HI, the increases in weight remained below those of the Sham group. These findings are consistent with previous work showing that $\mathrm{HI}$ insults impair somatic growth (Balduini, et al., 2001, Fan, et al., 2005, Lubics, et al., 2005). However, the rate of weight gain in males treated with IAIPs immediately after HI was greater than in those treated with placebo. Improved growth after treatment with IAIPs or with the light chain (bikunin) has not been previously reported. The mechanism(s) underlying improved growth cannot be determined from the current study because we did not measure milk intake or energy metabolism/balance in the neonatal rats. However, possible causes include increased intake because of improved general health, improved neurological integrity after treatment with IAIPs, improved energy balance, and/or decreased activity. Although we did not measure body composition in our neonatal rats exposed to $\mathrm{HI}$ with or without IAIP treatment and therefore cannot determine whether the increased weight gain in the IAIP-treated groups represented greater lean body or fat mass, inspection of the neonatal rats at necropsy suggested that their body habitus appeared similar to their non-IAIP-treated litter mates.

Our findings of sex related differences in neuroprotection were not expected. All of the pathological analysis and iimmunohistochemical detection was performed without knowledge of the study group or animal sex. However, based upon the histopathological scoring, cell death results and CNPase immunohistochemical detections, the overall 
favorable neuroprotective effects of IAIPs in the males and not in the females are important because of the well-known relative 'male disadvantage.' Males are predisposed to less favorable survival and neurodevelopmental outcomes compared with female infants (Vohr, et al., 2005). The neuroprotective effects of treatment with IAIPs on the histopathological scores (Fig. 5), regional pathology (Fig. 4), cell death (Fig. 7 and 8) and CNPase immunohistochemical detection (Fig. 10) suggest that this treatment is could be more efficacious in the more-at-risk male subjects after HI. However, as indicated above, these analyses were performed on similar brain sections in the same groups of rats and differ from our results showing equivalent reductions in infarct volumes after treatment with IAIPs in both males and females (Fig. 6). Nonetheless, previous work has also demonstrated sexrelated differential effects in neuroprotection favoring alternatively males or females (Dong, et al., 2018, Hill and Fitch, 2012, Jia, et al., 2011, Netto, et al., 2017, Uluc, et al., 2013). Previous work has suggested that these sex differences could have implications for sexspecific neuroprotection (Hill and Fitch, 2012). Progesterone treatment has been shown to be neuroprotective in male but not female neonatal rats after exposure to HI (Dong, et al., 2018) and calcium-permeable transient receptor potential M2 (TRPM2) ion channel inhibitors also preferentially protect male neurons and brain against ischemia suggesting that TRPM2 inhibitors may be more protective against ischemic injury in male subjects (Jia, et al., 2011). On the other hand, a TrkB agonist protects against HI-induced hippocampal neuronal death, white matter injury, and improves neurological function more in females than in males (Uluc, et al., 2013). Therefore, neuroprotective agents may have differential effects on males and females. Nonetheless, IAIPs appear to have similar neuroprotective effects in males and females based upon our measures of infarct volumes, which examines injury to the entire brain and is most likely more sensitive than histopathological or histochemical analysis of one brain section.

There are several other limitations to our study and opportunities for future research. It is possible that higher doses of IAIPs or an increased number of doses could have had greater neuroprotective effects. It would also be important to examine the effects of delayed treatment with IAIPs on measures of infarct volume to determine if the delayed IAIP treatment diminishes the $\mathrm{HI}$ related brain volume loss. Moreover, staining for ApopTag ${ }^{+}$ cells with and without staining for markers of astrocytes, microglia and oligodendrocytes was not performed in current study. Thus, we have not identified the specific cell types that were also ApopTag ${ }^{+}$, which would be important to investigate in future studies. Another important area to consider would be the potential protective effects of IAIPs on blood-brain barrier dysfunction after HI.

\section{Conclusions}

Systemic treatment with human blood-derived IAIPs administered immediately after HI attenuate histopathological injury in the cortex, hippocampus, caudate/putamen, cingulate and thalamus, attenuates HI-related increases in neuronal and non-neuronal cell death and white matter damage in male, but not in female neonatal rats. Systemic treatment with human blood-derived IAIPs administered immediately after HI reduces infarct volumes in both male and female rats. Immediate treatment with IAIPs after HI also improves the weight gain in male neonatal rats after HI. Delayed treatment $6 \mathrm{~h}$ after HI spares brain 
weights in male rats compared with placebo-treated rats exposed to HI. Therefore, human blood-derived IAIPs are neuroprotective after exposure to $\mathrm{HI}$ in neonatal rats.

\section{Acknowledgements}

We would like to thank Sunil Shaw, Ph.D. (COBRE Research Core, Women \& Infants Hospital of Rl) and Virginia Hovanesian, B.Sc. (Core Research Laboratories, Rhode Island Hospital) who provided expert assistance with image acquisition and analyses.

Funding

Research reported in this publication was supported by the National Institute of General Medical Sciences of the National Institutes of Health under the following award numbers: Institutional Development Award (IDeA) from the National Institute of General Medical Sciences of the National Institutes of Health under grant number P30GM114750, National Institutes of Health 1R21NS095130, 1R21NS096525 and R44 NS084575. The authors assume all responsibility for the study, and assert that the contents herein do not represent the National Institutes of Health's official views.
Abbreviations
HI
Hypoxia-Ischemia
IAIP
Inter-alpha Inhibitor Protein
PL
placebo

\section{References}

Abe H, Sugino N, Matsuda T, Kanamaru T, Oyanagi S, and Mori H, 1996 Effect of ulinastatin on delayed neuronal death in the gerbil hippocampus. Masui 45, 38-43. [PubMed: 8865723]

Adhikari S, and Rao KS, 2017 Neurodevelopmental outcome of term infants with perinatal asphyxia with hypoxic ischemic encephalopathy stage II. Brain Dev 39, 107-111. [PubMed: 27697304]

Andersen SL, 2003 Trajectories of brain development: point of vulnerability or window of opportunity? Neurosci Biobehav Rev 27, 3-18. [PubMed: 12732219]

Andine P, Thordstein M, Kjellmer I, Nordborg C, Thiringer K, Wennberg E, and Hagberg H, 1990 Evaluation of brain damage in a rat model of neonatal hypoxic-ischemia. J Neurosci Methods 35, 253-260. [PubMed: 2084395]

Balduini W, De Angelis V, Mazzoni E, and Cimino M, 2001 Simvastatin protects against long-lasting behavioral and morphological consequences of neonatal hypoxic/ischemic brain injury. Stroke 32, 2185-2191. [PubMed: 11546915]

Bockhorst KH, Narayana PA, Liu R, Ahobila-Vijjula P, Ramu J, Kamel M, Wosik J, Bockhorst T, Hahn K, Hasan KM, and Perez-Polo JR, 2008 Early postnatal development of rat brain: in vivo diffusion tensor imaging. J Neurosci Res 86, 1520-1528. [PubMed: 18189320]

Bost F, Diarra-Mehrpour M, and Martin JP, 1998 Inter-alpha-trypsin inhibitor proteoglycan family--a group of proteins binding and stabilizing the extracellular matrix. Eur J Biochem 252, 339-346. [PubMed: 9546647]

Boxenbaum H, 1982 Interspecies scaling, allometry, physiological time, and the ground plan of pharmacokinetics. J Pharmacokinet Biopharm 10, 201-227. [PubMed: 7120049]

Brown AW, and Brierley JB, 1972 Anoxic-ischaemic cell change in rat brain light microscopic and fine-structural observations. J Neurol Sci 16, 59-84. [PubMed: 5034855]

Businaro R, Leali FM, De Renzis G, Pompili E, Pagliari G, Menghi G, and Fumagalli L, 1992 Interalpha-trypsin inhibitor-related immunoreactivity in human tissues and body fluids. Cell Mol Biol 38, 463-471. [PubMed: 1379887]

Chaaban H, Shin M, Sirya E, Lim YP, Caplan M, and Padbury JF, 2010 Inter-alpha inhibitor protein level in neonates predicts necrotizing enterocolitis. J Pediatr 157, 757-761. [PubMed: 20955849] 
Chaaban H, Singh K, Huang J, Siryaporn E, Lim YP, and Padbury JF, 2009 The role of inter-alpha inhibitor proteins in the diagnosis of neonatal sepsis. J Pediatr 154, 620-622.e621. [PubMed: 19324226]

Chen HM, Huang HS, Ruan L, He YB, and Li XJ, 2014 Ulinastatin attenuates cerebral ischemiareperfusion injury in rats. Int J Clin Exp Med 7, 1483-1489. [PubMed: 24995117]

Chen X, Zhang J, Kim B, Jaitpal S, Meng SS, Adjepong K, Imamura S, Wake H, Nishibori M, Stopa EG, and Stonestreet BS, 2019 High-mobility group box-1 translocation and release after hypoxic ischemic brain injury in neonatal rats. Exp Neurol 311, 1-14. [PubMed: 30217406]

Cho YS, Shin MS, Ko IG, Kim SE, Kim CJ, Sung YH, Yoon HS, and Lee BJ, 2015 Ulinastatin inhibits cerebral ischemia-induced apoptosis in the hippocampus of gerbils. Mol Med Rep 12, 1796-1802. [PubMed: 25891426]

Cotten CM, and Shankaran S, 2010 Hypothermia for hypoxic-ischemic encephalopathy. Expert Rev Obstet Gynecol 5, 227-239. [PubMed: 20625441]

Cowan WM, 1979 The development of the brain. Sci Am 241, 113-133. [PubMed: 493917]

Dobbing J, and Sands J, 1979 Comparative aspects of the brain growth spurt. Early Hum Dev 3, 7983. [PubMed: 118862]

Dong S, Zhang Q, Kong D, Zhou C, Zhou J, Han J, Zhou Y, Jin G, Hua X, Wang J , and Hua F, 2018 Gender difference in the effect of progesterone on neonatal hypoxic/ischemic brain injury in mouse. Exp Neurol 306, 190-198. [PubMed: 29772244]

Fan L-W, Lin S, Pang Y, Lei M, Zhang F, Rhodes PG, and Cai Z, 2005 Hypoxia-ischemia induced neurological dysfunction and brain injury in the neonatal rat. Behavioural Brain Research 165, 8090. [PubMed: 16140403]

Fan LW, Lin S, Pang Y, Lei M, Zhang F, Rhodes PG, and Cai Z, 2005 Hypoxia-ischemia induced neurological dysfunction and brain injury in the neonatal rat. Behav Brain Res 165, 80-90. [PubMed: 16140403]

Feng M, Shu Y, Yang Y, Zheng X, Li R, Wang Y, Dai Y, Qiu W, Lu Z, and Hu X, 2014 Ulinastatin attenuates experimental autoimmune encephalomyelitis by enhancing anti-inflammatory responses. Neurochem Int 64, 64-72. [PubMed: 24274996]

Ferriero DM, 2004 Neonatal brain injury. N Engl J Med 351, 1985-1995. [PubMed: 15525724]

Fries E, and Blom AM, 2000 Bikunin--not just a plasma proteinase inhibitor. Int J Biochem Cell Biol 32, 125-137. [PubMed: 10687949]

Garantziotis S, Hollingsworth JW, Ghanayem RB, Timberlake S, Zhuo L, Kimata K, and Schwartz DA, 2007 Inter-alpha-trypsin inhibitor attenuates complement activation and complement-induced lung injury. J Immunol 179, 4187-4192. [PubMed: 17785858]

Gaudet CM, Lim YP, Stonestreet BS, and Threlkeld SW, 2016 Effects of age, experience and interalpha inhibitor proteins on working memory and neuronal plasticity after neonatal hypoxiaischemia. Behav Brain Res 302, 88-99. [PubMed: 26778784]

Graham EM, Ruis KA, Hartman AL, Northington FJ, and Fox HE, 2008 A systematic review of the role of intrapartum hypoxia-ischemia in the causation of neonatal encephalopathy. Am J Obstet Gynecol 199, 587-595. [PubMed: 19084096]

Gunn AJ, Gunn TR, de Haan HH, Williams CE, and Gluckman PD, 1997 Dramatic neuronal rescue with prolonged selective head cooling after ischemia in fetal lambs. The Journal of Clinical Investigation 99, 248-256. [PubMed: 9005993]

Hagberg H, Mallard C, Ferriero DM, Vannucci SJ, Levison SW, Vexler ZS, and Gressens P, 2015 The role of inflammation in perinatal brain injury. Nat Rev Neurol 11, 192-208. [PubMed: 25686754]

Hill CA, and Fitch RH, 2012 Sex differences in mechanisms and outcome of neonatal hypoxiaischemia in rodent models: implications for sex-specific neuroprotection in clinical neonatal practice. Neurol Res Int 2012, 867531. [PubMed: 22474588]

Hoque N, Sabir H, Maes E, Bishop S, and Thoresen M, 2014 Validation of a neuropathology score using quantitative methods to evaluate brain injury in a pig model of hypoxia ischaemia. $\mathrm{J}$ Neurosci Methods 230, 30-36. [PubMed: 24747875]

Hu CL, Xia JM, Cai J, Li X, Liao XX, Li H, Zhan H, Dai G, and Jing XL, 2013 Ulinastatin attenuates oxidation, inflammation and neural apoptosis in the cerebral cortex of adult rats with ventricular

Exp Neurol. Author manuscript; available in PMC 2020 July 01. 
fibrillation after cardiopulmonary resuscitation. Clinics (Sao Paulo) 68, 1231-1238. [PubMed: 24141840]

Jia J, Verma S, Nakayama S, Quillinan N, Grafe MR, Hurn PD, and Herson PS, 2011 Sex differences in neuroprotection provided by inhibition of TRPM2 channels following experimental stroke. $\mathrm{J}$ Cereb Blood Flow Metab 31, 2160-2168. [PubMed: 21587268]

Jiang XM, Hu JH, Wang LL, Ma C, Wang X, and Liu XL, 2016 Effects of ulinastatin on global ischemia via brain pro-inflammation signal. Transl Neurosci 7, 158-163. [PubMed: 28123836]

Klahr AC, Nadeau CA, and Colbourne F, 2017 Temperature Control in Rodent Neuroprotection Studies: Methods and Challenges. Ther Hypothermia Temp Manag 7, 42-49. [PubMed: 27327871]

Kobayashi H, 2006 Endogenous anti-inflammatory substances, inter-alpha-inhibitor and bikunin. Biol Chem 387, 1545-1549. [PubMed: 17132099]

Koga Y, Fujita M, Tsuruta R, Koda Y, Nakahara T, Yagi T, Aoki T, Kobayashi C, Izumi T, Kasaoka S, Yuasa M, and Maekawa T, 2010 Urinary trypsin inhibitor suppresses excessive superoxide anion radical generation in blood, oxidative stress, early inflammation, and endothelial injury in forebrain ischemia/reperfusion rats. Neurol Res 32, 925-932. [PubMed: 20223106]

Li X, Su L, Zhang X, Zhang C, Wang L, Li Y, Zhang Y, He T, Zhu X, and Cui L, 2017 Ulinastatin downregulates TLR4 and NF-kB expression and protects mouse brains against ischemia/ reperfusion injury. Neurol Res 39, 367-373. [PubMed: 28191863]

Li XF, Zhang XJ, Zhang C, Wang LN, Li YR, Zhang Y, He TT, Zhu XY, Cui LL, and Gao BL, 2017 Ulinastatin protects brain against cerebral ischemia/reperfusion injury through inhibiting MMP-9 and alleviating loss of ZO-1 and occludin proteins in mice. Exp Neurol 302, 68-74. [PubMed: 29291404]

Ligon KL, Fancy SP, Franklin RJ, and Rowitch DH, 2006 Olig gene function in CNS development and disease. Glia 54, 1-10. [PubMed: 16652341]

Lim YP, 2013 ProThera Biologics, Inc.: A Novel Immunomodulator and Biomarker for LifeThreatening Diseases. R I Med J (2013) 96, 16-18.

Lim YP, Josic D, Callanan H, Brown J, and Hixson DC, 2005 Affinity purification and enzymatic cleavage of inter-alpha inhibitor proteins using antibody and elastase immobilized on CIM monolithic disks. J Chromatogr A 1065, 39-43. [PubMed: 15782948]

Liu M, Shen J, Zou F, Zhao Y, Li B, and Fan M, 2017 Effect of ulinastatin on the permeability of the blood-brain barrier on rats with global cerebral ischemia/reperfusion injury as assessed by MRI. Biomed Pharmacother 85, 412-417. [PubMed: 27916423]

Lubics A, Reglodi D, Tamas A, Kiss P, Szalai M, Szalontay L, and Lengvari I, 2005 Neurological reflexes and early motor behavior in rats subjected to neonatal hypoxic-ischemic injury. Behav Brain Res 157, 157-165. [PubMed: 15617782]

Lundgaard I, Luzhynskaya A, Stockley JH, Wang Z, Evans KA, Swire M, Volbracht K, Gautier HOB, Franklin RJM, ffrench-Constant C, Attwell D, and Káradóttir RT, 2013 Neuregulin and BDNF Induce a Switch to NMDA Receptor-Dependent Myelination by Oligodendrocytes. PLoS Biology $11,1-18$.

Martinez-Biarge M, Bregant T, Wusthoff CJ, Chew AT, Diez-Sebastian J, Rutherford MA, and Cowan FM, 2012 White matter and cortical injury in hypoxic-ischemic encephalopathy: antecedent factors and 2-year outcome. J Pediatr 161, 799-807. [PubMed: 22682614]

McAdams RM, and Juul SE, 2012 The role of cytokines and inflammatory cells in perinatal brain injury. Neurol Res Int 2012, 561494. [PubMed: 22530124]

Mecha M, Feliú A, Carrillo-Salinas FJ, Mestre L, and Guaza C, 2013 Mobilization of progenitors in the subventricular zone to undergo oligodendrogenesis in the Theiler's virus model of multiple sclerosis: Implications for remyelination at lesions sites. Experimental Neurology 250, 348-352. [PubMed: 24148569]

Netto CA, Sanches E, Odorcyk FK, Duran-Carabali LE, and Weis SN, 2017 Sex-dependent consequences of neonatal brain hypoxia-ischemia in the rat. J Neurosci Res 95, 409-421. [PubMed: 27870406]

Northington FJ, Chavez-Valdez R, and Martin LJ, 2011 Neuronal cell death in neonatal hypoxiaischemia. Ann Neurol 69, 743-758. [PubMed: 21520238] 
Northington FJ, Ferriero DM, Flock DL, and Martin LJ, 2001 Delayed neurodegeneration in neonatal rat thalamus after hypoxia-ischemia is apoptosis. J Neurosci 21, 1931-1938. [PubMed: 11245678]

Northington FJ, Ferriero DM, Graham EM, Traystman RJ, and Martin LJ, 2001 Early Neurodegeneration after Hypoxia-Ischemia in Neonatal Rat Is Necrosis while Delayed Neuronal Death Is Apoptosis. Neurobiol Dis 8, 207-219. [PubMed: 11300718]

Northington FJ, Ferriero DM, and Martin LJ, 2001 Neurodegeneration in the thalamus following neonatal hypoxia-ischemia is programmed cell death. Dev Neurosci 23, 186-191. [PubMed: 11598318]

Northington FJ, Graham EM, and Martin LJ, 2005 Apoptosis in perinatal hypoxic-ischemic brain injury: how important is it and should it be inhibited? Brain Res Brain Res Rev 50, 244-257. [PubMed: 16216332]

Oakden E, Chiswick M, Rothwell N, and Loddick S, 2002 The influence of litter size on brain damage caused by hypoxic-ischemic injury in the neonatal rat. Pediatr Res 52, 692-696. [PubMed: 12409515]

Opal SM, Lim YP, Cristofaro P, Artenstein AW, Kessimian N, Delsesto D, Parejo N, Palardy JE, and Siryaporn E, 2011 Inter-alpha inhibitor proteins: a novel therapeutic strategy for experimental anthrax infection. Shock 35, 42-44. [PubMed: 20523269]

Osredkar D, Thoresen M, Maes E, Flatebo T, Elstad M, and Sabir H, 2014 Hypothermia is not neuroprotective after infection-sensitized neonatal hypoxic-ischemic brain injury. Resuscitation 85 , 567-572. [PubMed: 24361672]

Paxinos G, and Watson C, 2017 The Rat Brain in Stereotaxic Coordinates: Compact. Elsevier Science.

Petersson KH, Pinar H, Stopa EG, Faris RA, Sadowska GB, Hanumara RC, and Stonestreet BS, 2002 White matter injury after cerebral ischemia in ovine fetuses. Pediatr Res 51, 768-776. [PubMed: 12032276]

Potempa J, Kwon K, Chawla R, and Travis J, 1989 Inter-alpha-trypsin inhibitor. Inhibition spectrum of native and derived forms. J Biol Chem 264, 15109-15114. [PubMed: 2475494]

Qiao L, Fu J, Xue X, Shi Y, Yao L, Huang W, Li J, Zhang D, Liu N, Tong X, Du Y, and Pan Y, 2018 Neuronalinjury and roles of apoptosis and autophagy in a neonatal rat model of hypoxia-ischemiainduced periventricular leukomalacia. Molecular Medicine Reports 17, 5940-5949. [PubMed: 29436652]

Reddy K, Mallard C, Guan J, Marks K, Bennet L, Gunning M, Gunn A, Gluckman P, and Williams C, 1998 Maturational change in the cortical response to hypoperfusion injury in the fetal sheep. Pediatr Res 43, 674-682. [PubMed: 9585015]

Rice JE, Vannucci RC, and Brierley JB, 1981 The influence of immaturity on hypoxic-ischemic brain damage in the rat. Annals of Neurology 9, 131-141. [PubMed: 7235629]

Rumajogee P, Bregman T, Miller SP, Yager JY, and Fehlings MG, 2016 Rodent Hypoxia-Ischemia Models for Cerebral Palsy Research: A Systematic Review. Front Neurol 7, 57. [PubMed: 27199883]

Salier JP, Rouet P, Raguenez G, and Daveau M, 1996 The inter-alpha-inhibitor family: from structure to regulation. Biochem J 315 ( Pt 1), 1-9. [PubMed: 8670091]

Sawada M, Alkayed NJ, Goto S, Crain BJ, Traystman RJ, Shaivitz A, Nelson RJ, and Hurn PD, 2000 Estrogen receptor antagonist ICI182,780 exacerbates ischemic injury in female mouse. J Cereb Blood Flow Metab 20, 112-118. [PubMed: 10616799]

Schindelin J, Arganda-Carreras I, Frise E, Kaynig V, Longair M, Pietzsch T, Preibisch S, Rueden C, Saalfeld S, Schmid B, Tinevez JY, White DJ, Hartenstein V, Eliceiri K, Tomancak P, and Cardona A, 2012 Fiji: an open-source platform for biological-image analysis. Nat Methods 9, 676-682. [PubMed: 22743772]

Semple BD, Blomgren K, Gimlin K, Ferriero DM, and Noble-Haeusslein LJ, 2013 Brain development in rodents and humans: Identifying benchmarks of maturation and vulnerability to injury across species. Prog Neurobiol 106-107, 1-16. [PubMed: 23583307]

Shah DK, Doyle LW, Anderson PJ, Bear M, Daley AJ, Hunt RW, and Inder TE, 2008 Adverse neurodevelopment in preterm infants with postnatal sepsis or necrotizing enterocolitis is mediated by white matter abnormalities on magnetic resonance imaging at term. J Pediatr 153, 170-175, 175 e171. [PubMed: 18534228] 
Shankaran S, Laptook AR, Ehrenkranz RA, Tyson JE, McDonald SA, Donovan EF, Fanaroff AA, Poole WK, Wright LL, Higgins RD, Finer NN, Carlo WA, Duara S, Oh W, Cotten CM, Stevenson DK, Stoll BJ, Lemons JA, Guillet R, Jobe AH, National Institute of Child, H., and Human Development Neonatal Research, N., 2005 Whole-body hypothermia for neonates with hypoxicischemic encephalopathy. N Engl J Med 353, 1574-1584. [PubMed: 16221780]

Shindo A, Liang AC, Maki T, Miyamoto N, Tomimoto H, Lo EH, and Arai K, 2016 Subcortical ischemic vascular disease: Roles of oligodendrocyte function in experimental models of subcortical white-matter injury. 36, 187-198.

Shu Y, Yang Y, Qiu W, Lu Z, Li Y, Bao J, Feng M, and Hu X, 2011 Neuroprotection by ulinastatin in experimental autoimmune encephalomyelitis. Neurochem Res 36, 1969-1977. [PubMed: 21667278]

Singh AV, Palladino MA, Lloyd GK, Potts BC, Chauhan D, and Anderson KC, 2010 Pharmacodynamic and efficacy studies of the novel proteasome inhibitor NPI-0052 (marizomib) in a human plasmacytoma xenograft murine model. Br J Haematol 149, 550-559. [PubMed: 20331453]

Sjoberg EM, Blom A, Larsson BS, Alston-Smith J, Sjoquist M, and Fries E, 1995 Plasma clearance of rat bikunin: evidence for receptor-mediated uptake. Biochem J 308 ( Pt 3), 881-887. [PubMed: 8948446]

Spasova MS, Sadowska GB, Threlkeld SW, Lim YP, and Stonestreet BS, 2014 Ontogeny of inter-alpha inhibitor proteins in ovine brain and somatic tissues. Exp Biol Med (Maywood) 239, 724-736. [PubMed: 24728724]

Stoll BJ, Hansen NI, Adams-Chapman I, Fanaroff AA, Hintz SR, Vohr B, and Higgins RD, 2004 Neurodevelopmental and growth impairment among extremely low-birth-weight infants with neonatal infection. Jama. 292, 2357-2365. [PubMed: 15547163]

Swanson RA, Morton MT, Tsao-Wu G, Savalos RA, Davidson C, and Sharp FR, 1990 A semiautomated method for measuring brain infarct volume. J Cereb Blood Flow Metab 10, 290 293. [PubMed: 1689322]

Threlkeld SW, Gaudet CM, La Rue ME, Dugas E, Hill CA, Lim YP, and Stonestreet BS, 2014 Effects of inter-alpha inhibitor proteins on neonatal brain injury: Age, task and treatment dependent neurobehavioral outcomes. Exp Neurol 261, 424-433. [PubMed: 25084519]

Threlkeld SW, Lim YP, La Rue M, Gaudet C, and Stonestreet BS, 2017 Immunomodulator inter-alpha inhibitor proteins ameliorate complex auditory processing deficits in rats with neonatal hypoxicischemic brain injury. Brain Behav Immun 64, 173-179. [PubMed: 28286301]

Uluc K, Kendigelen P, Fidan E, Zhang L, Chanana V, Kintner D, Akture E, Song C, Ye K, Sun D, Ferrazzano P, and Cengiz P, 2013 TrkB receptor agonist 7, 8 dihydroxyflavone triggers profound gender-dependent neuroprotection in mice after perinatal hypoxia and ischemia. CNS Neurol Disord Drug Targets 12, 360-370. [PubMed: 23469848]

Vannucci RC, Towfighi J, and Vannucci SJ, 2004 Secondary energy failure after cerebral hypoxiaischemia in the immature rat. J Cereb Blood Flow Metab 24, 1090-1097. [PubMed: 15529009]

Vohr BR, Wright LL, Poole WK, and McDonald SA, 2005 Neurodevelopmental outcomes of extremely low birth weight infants <32 weeks' gestation between 1993 and 1998. Pediatrics 116, 635-643. [PubMed: 16143580]

Volpe J, 1995 Hypoxic-ischemic encephalopathy In: Neurology of the Newborn. WB Saunders, Philadelphia, PA, pp 260-313.

Walsh MC, Kliegman RM, and Hack M, 1989 Severity of necrotizing enterocolitis: influence on outcome at 2 years of age. Pediatrics 84, 808-814. [PubMed: 2797976]

Wang X, Hellgren G, Lofqvist C, Li W, Hellstrom A, Hagberg H, and Mallard C, 2009 White matter damage after chronic subclinical inflammation in newborn mice. J Child Neurol 24, 1171-1178. [PubMed: 19745089]

Wang X, Xue Q, Yan F, Li L, Liu J, Li S, and Hu S, 2013 Ulinastatin as a neuroprotective and antiinflammatory agent in infant piglets model undergoing surgery on hypothermic low-flow cardiopulmonary bypass. Paediatr Anaesth 23, 209-216. [PubMed: 23384299] 
Wu R, Cui X, Lim YP, Bendelja K, Zhou M, Simms HH, and Wang P, 2004 Delayed administration of human inter-alpha inhibitor proteins reduces mortality in sepsis. Crit Care Med 32, 1747-1752. [PubMed: 15286553]

Yang S, Lim YP, Zhou M, Salvemini P, Schwinn H, Josic D, Koo DJ, Chaudry IH, and Wang P, 2002 Administration of human inter-alpha-inhibitors maintains hemodynamic stability and improves survival during sepsis. Crit Care Med 30, 617-622. [PubMed: 11990925]

Yano T, Anraku S, Nakayama R, and Ushijima K, 2003 Neuroprotective effect of urinary trypsin inhibitor against focal cerebral ischemia-reperfusion injury in rats. Anesthesiology 98, 465-473. [PubMed: 12552207]

Zhang L, Nair A, Krady K, Corpe C, Bonneau RH, Simpson IA, and Vannucci SJ, 2004 Estrogen stimulates microglia and brain recovery from hypoxia-ischemia in normoglycemic but not diabetic female mice. J Clin Invest 113, 85-95. [PubMed: 14702112]

Zhu L, Zhuo L, Watanabe H, and Kimata K, 2008 Equivalent involvement of inter-alpha-trypsin inhibitor heavy chain isoforms in forming covalent complexes with hyaluronan. Connect Tissue Res 49, 48-55. [PubMed: 18293178] 


\section{Highlights}

- $\quad$ Neuroinflammation is a key mechanism underlying hypoxic-ischemic (HI) brain injury

- Inter-alpha inhibitor proteins (IAIPs) are immunomodulatory molecules

- $\quad$ IAIPs reduce HI-related neuronal and non-neuronal cell death in male neonatal rats

- $\quad$ IAIPs attenuate HI-related brain infarction volume in neonatal rats 


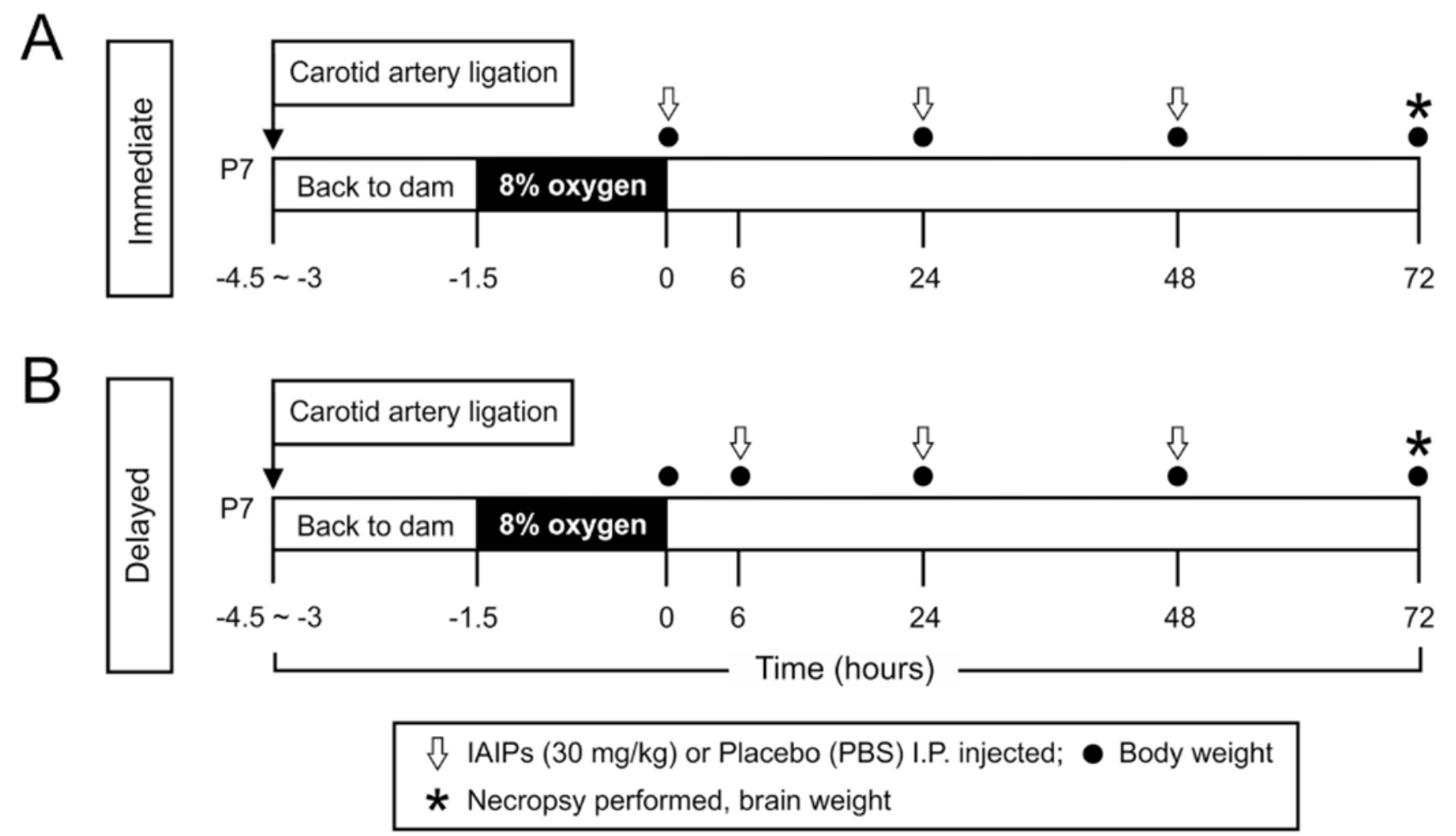

Fig. 1.

Study design. (A) The pups were returned to the dams for 1.5 to $3 \mathrm{~h}$ after right common carotid artery ligation. Thereafter, the pups were exposed to $8 \%$ oxygen with balanced nitrogen for $90 \mathrm{~min}$ at a constant temperature of $36^{\circ} \mathrm{C}$. Thirty $\mathrm{mg} / \mathrm{kg}$ of human blood-derived IAIPs or placebo were given intraperitoneally (I.P.) immediately, 24 and $48 \mathrm{~h}$ after termination of hypoxia. Body weight was measured immediately, 24, 48 and $72 \mathrm{~h}$ after hypoxia. Seventy-two $\mathrm{h}$ after hypoxia, a necropsy was performed and brain weight obtained. (B) Study design for delayed treatment. The procedures were the same as for the study design in A except that IAIPs or placebo were given 6, 24 and $48 \mathrm{~h}$ after the termination of hypoxia. Body weight was measured immediately, 6, 24, 48 and $72 \mathrm{~h}$ after hypoxia. Seventytwo $\mathrm{h}$ after hypoxia, an necropsy was performed and brain weight obtained. IAIP = InterAlpha Inhibitor proteins, PBS = phosphate buffered saline. 

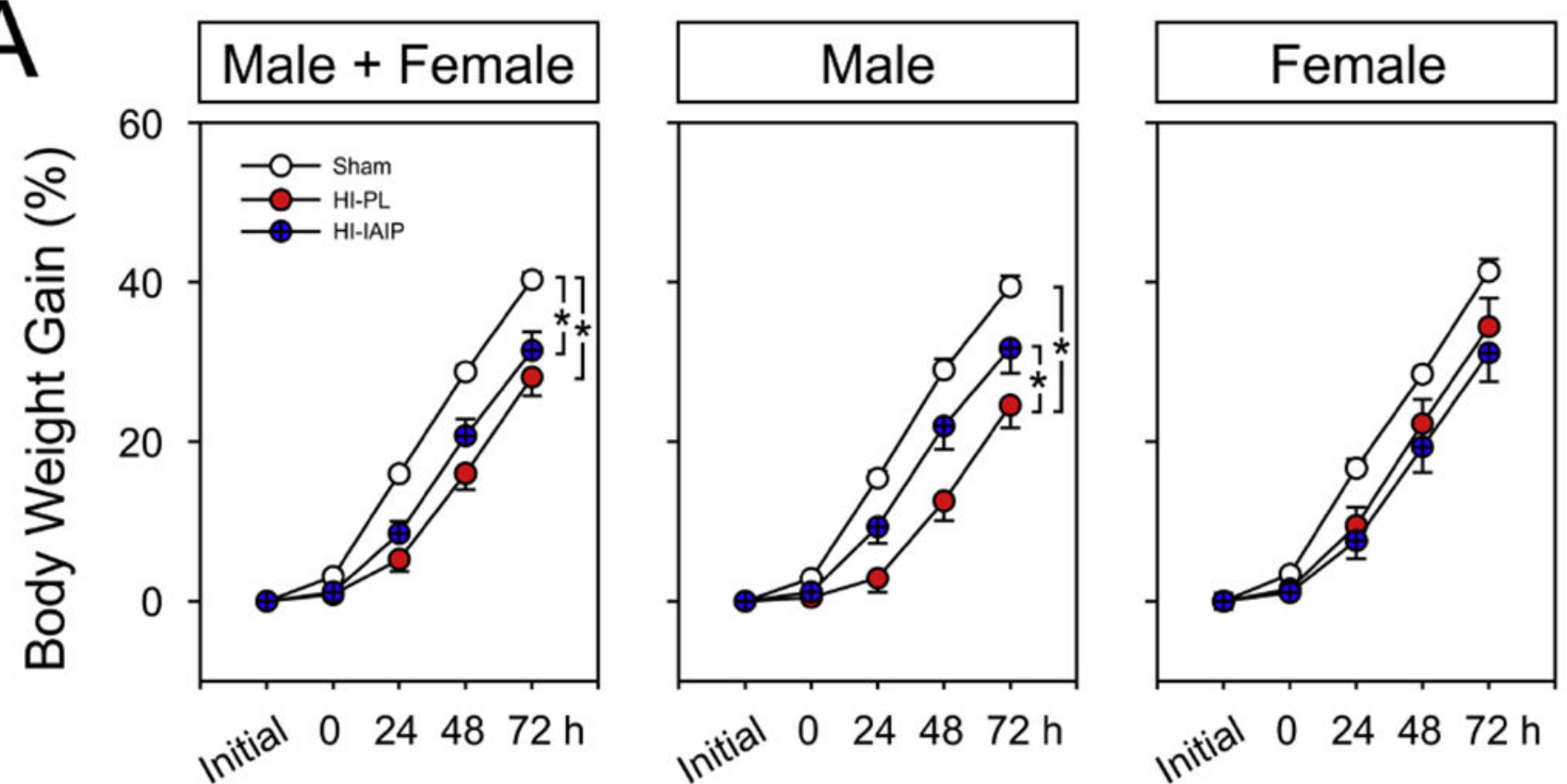

$\mathrm{B}$
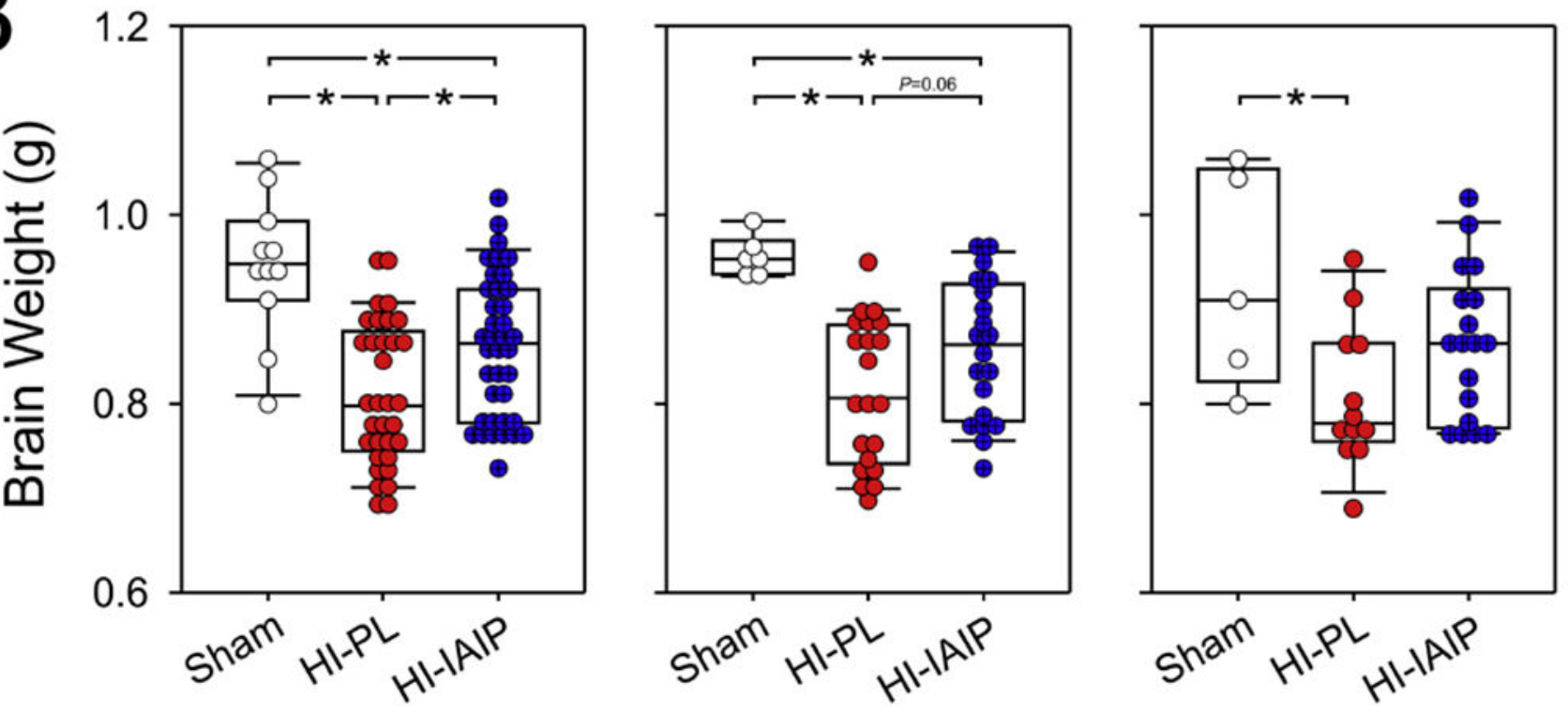

Fig. 2.

Body weight gain (\%) and brain weights (g) of the Sham, HI-PL and HI-IAIP groups after immediate treatment with IAIPs or placebo in neonatal rats exposed to HI brain injury. (A) Percent body weight gain on the y-axis plotted against study time in hours on the $\mathrm{x}$-axis for the males + females, males and females. Sham: male $n=6$, female $n=5$; HI-PL: male $n=$ 25, female $\mathrm{n}=14$; HI-IAIP: male $\mathrm{n}=23$, female $\mathrm{n}=20$. Values are mean \pm SEM. (B) Brain weights of the males + females, males, and females plotted as dot box plots showing the median, and $5^{\text {th }}$ and $95^{\text {th }}$ percentiles. Sham: male $n=6$, female $n=5$; HI-PL: male $n=21$, female $\mathrm{n}=12$; HI-IAIP: male $\mathrm{n}=20$, female $\mathrm{n}=18$. $* \mathrm{P}<0.05$. 

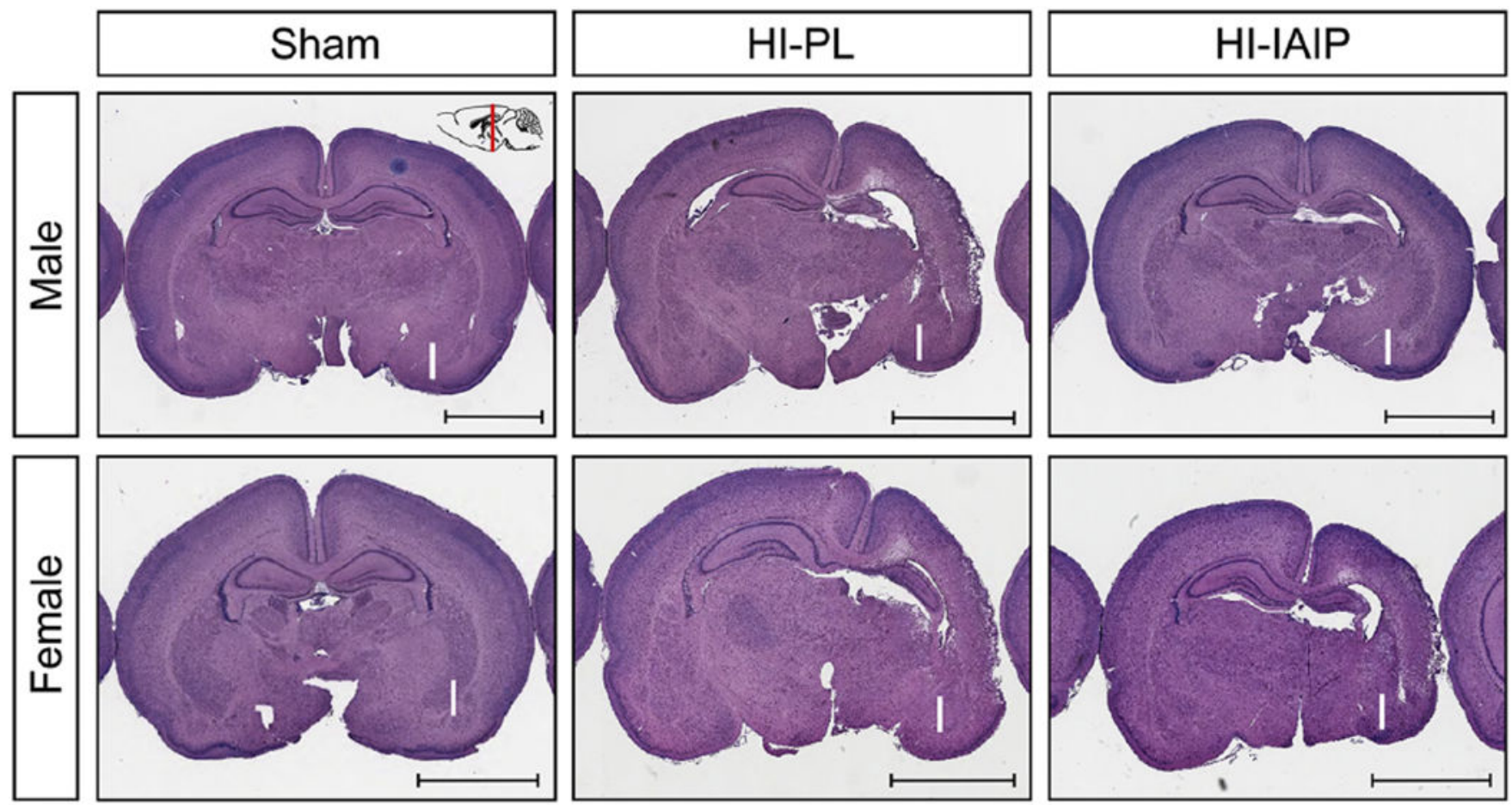

Fig. 3.

Representative coronal sections stained with LFB\&HE from the Sham, HI-PL and HI-IAIP males (top row) and females (bottom row) after immediate treatment with placebo or IAIPs. The "I" designates the side of the brain ipsilateral to the carotid artery ligation. Scale bar $=3$ $\mathrm{mm}$. 

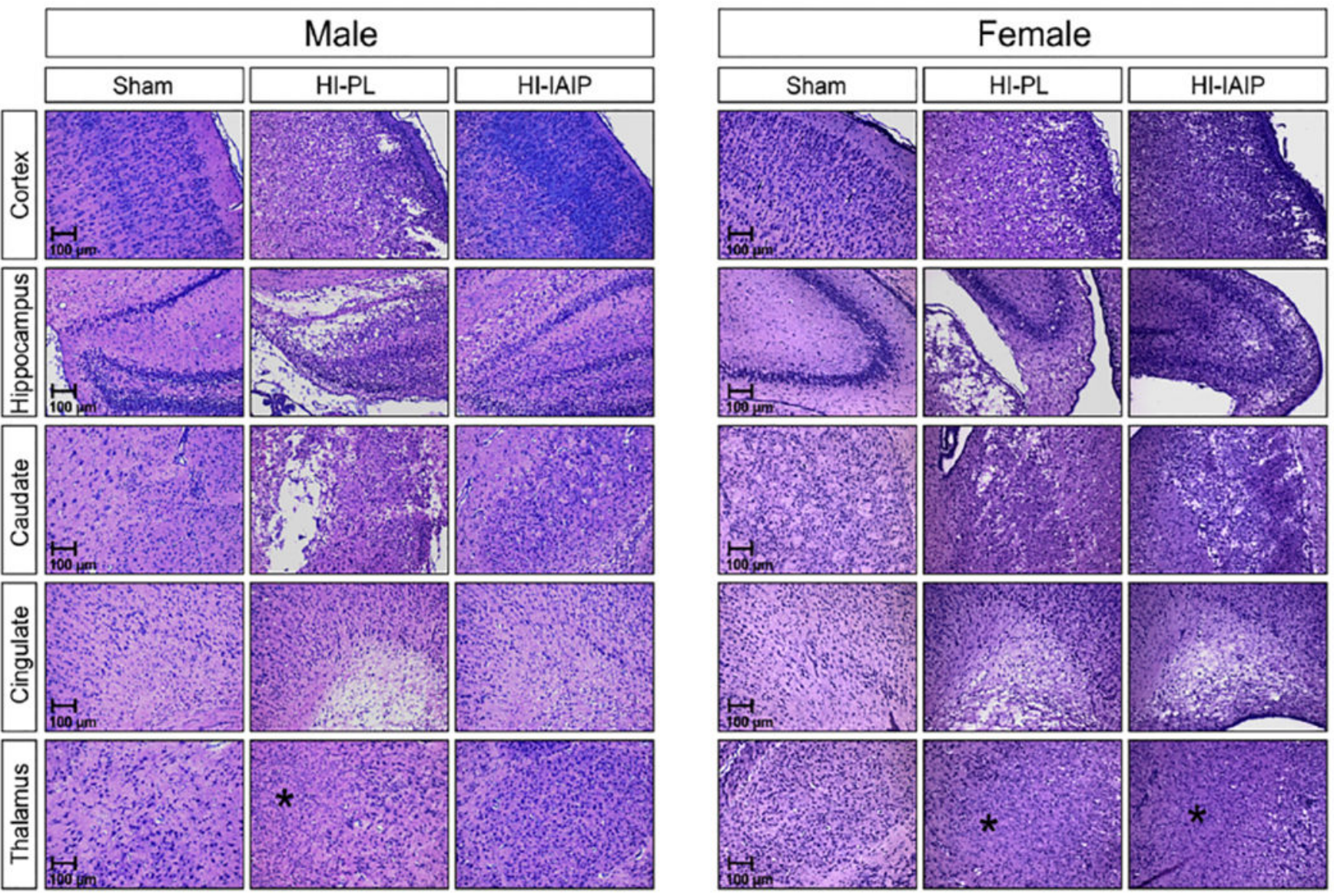

Fig. 4.

Representative sections stained with LFB\&HE of the parietal cortex, hippocampus, caudate/ putamen, cingulate and thalamus from the Sham, HI-PL, and HI-IAIP groups of male and female neonatal rats after immediate treatment with placebo or IAIPs. HI-related injury in the thalamus consisted of cystic and non-cystic infarction (see asterisks), neuronal loss, edema and reactive gliosis. Scale bars $100 \mu \mathrm{m}$. 

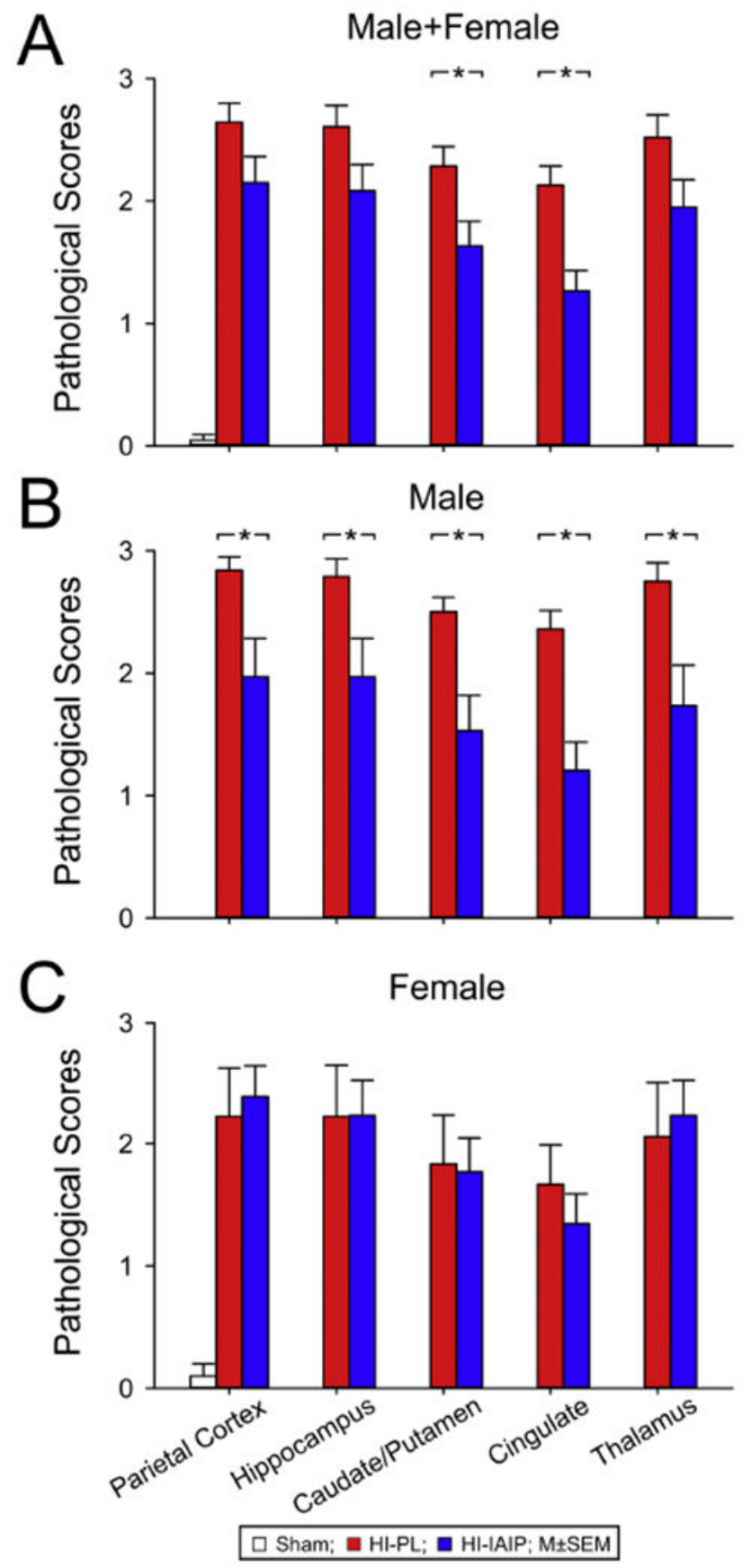

Fig. 5.

Pathological scores plotted on the y-axis for the parietal cortex, hippocampus, caudate/ putamen, cingulate and thalamus on the $\mathrm{x}$-axis for the (A) male + females, (B) males and (C) females in the Sham, HI-PL, and HI-IAIP groups after immediate treatment with placebo or IAIPs. Values are mean \pm SEM. $* P<0.05)$. Sham: male $n=6$, female $n=5$; HI-PL: male $n$ $=19$, female $n=9$; HI-IAIP: male $n=17$, female $n=13$. 
A
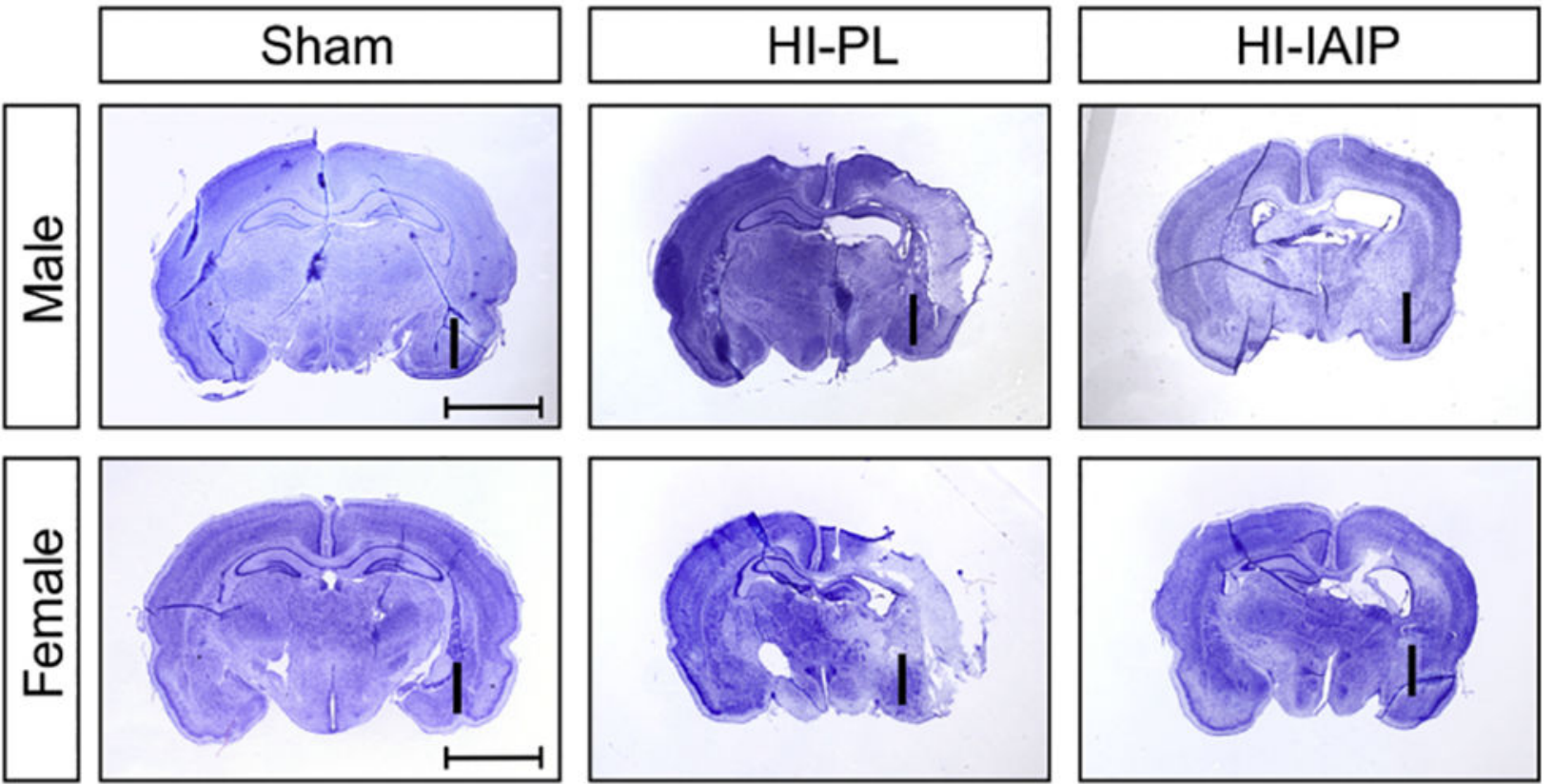

$\mathrm{B}$
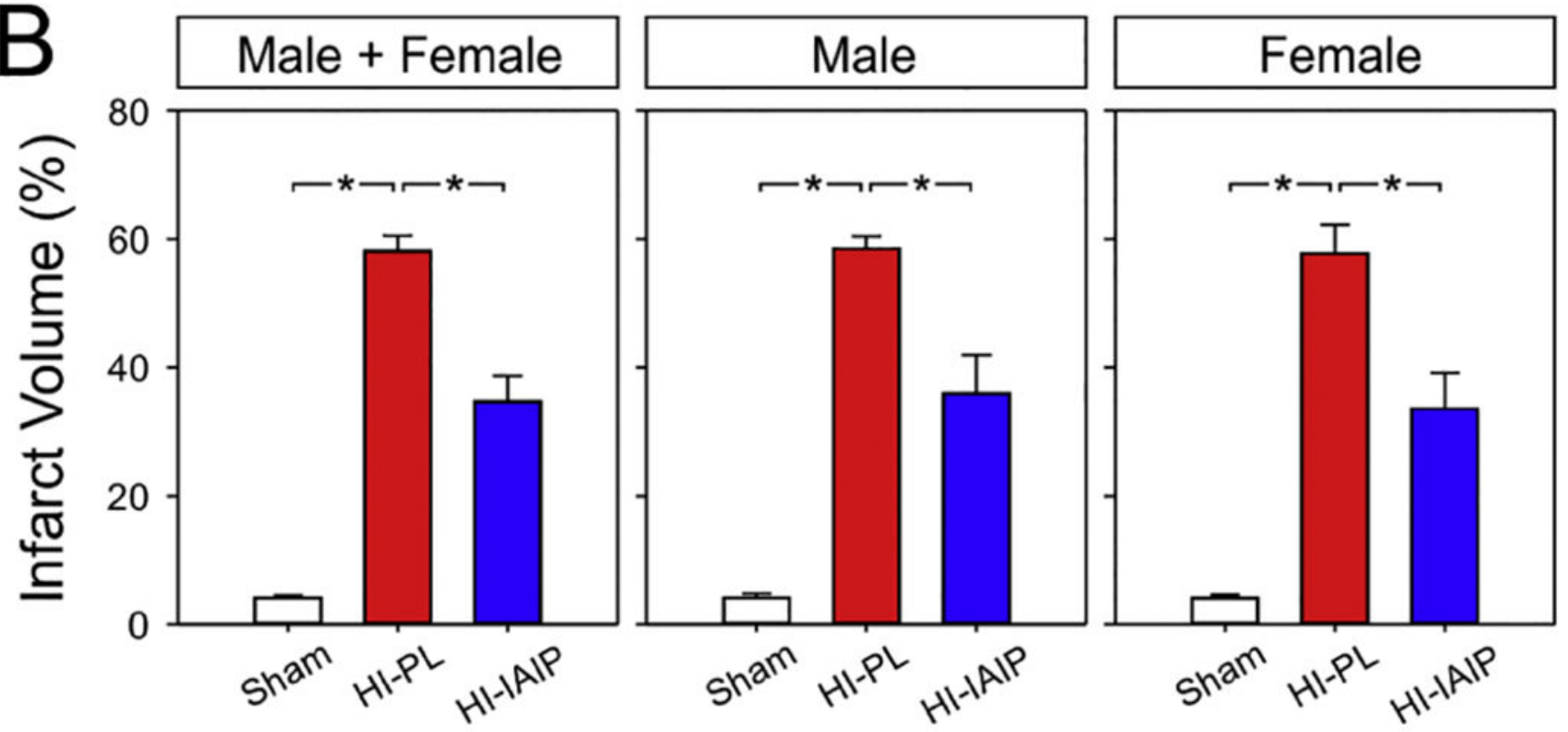

Fig. 6.

(A) Representative images of brain slices stained with cresyl violet 3 days after HI brain injury. The "I" designates the side of the brain ipsilateral to the carotid artery ligation. Compared to the HI-PL hemisphere, the infarct area in both males (top row) and females (bottom row) were reduced after IAIP treatment. (B) Percent infarct plotted on the y-axis for the Sham, HI-PL, and HI-IAIP groups on the $\mathrm{x}$-axis for males + females, males and females. Values are mean \pm SEM. $* P<0.05$. Sham: male $\mathrm{n}=12$, female $\mathrm{n}=5$; HI-PL: male $\mathrm{n}=8$, female $\mathrm{n}=9$; HI-IAIP: male $\mathrm{n}=11$, female $\mathrm{n}=12$. 

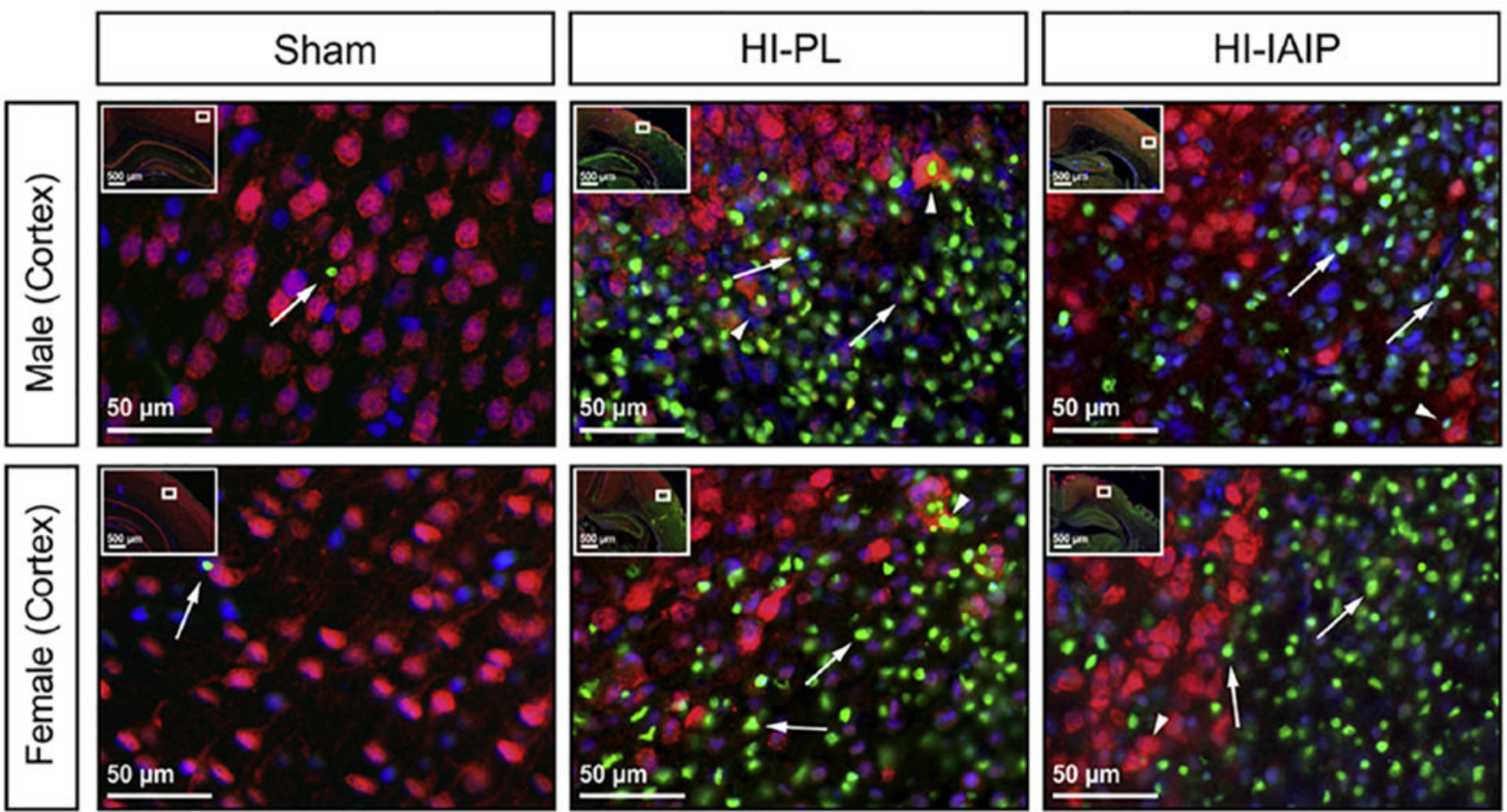

NeuN ApopTag DAPI

Fig. 7.

Representative immunohistochemical images containing NeuN- (red) and ApopTag-labeled (green) cells in the cerebral cortices of the Sham, HI-PL and HI-IAIP male (top row) and female (bottom row) neonatal rats. DAPI (blue) is utilized as a counterstain. The inset on each image identifies the area of the brain from which the image was taken. White arrows indicate ApopTag-labeled cells (ApopTag ${ }^{+}$). White arrowheads indicate ApopTag-positive and NeuN-positive cells. 

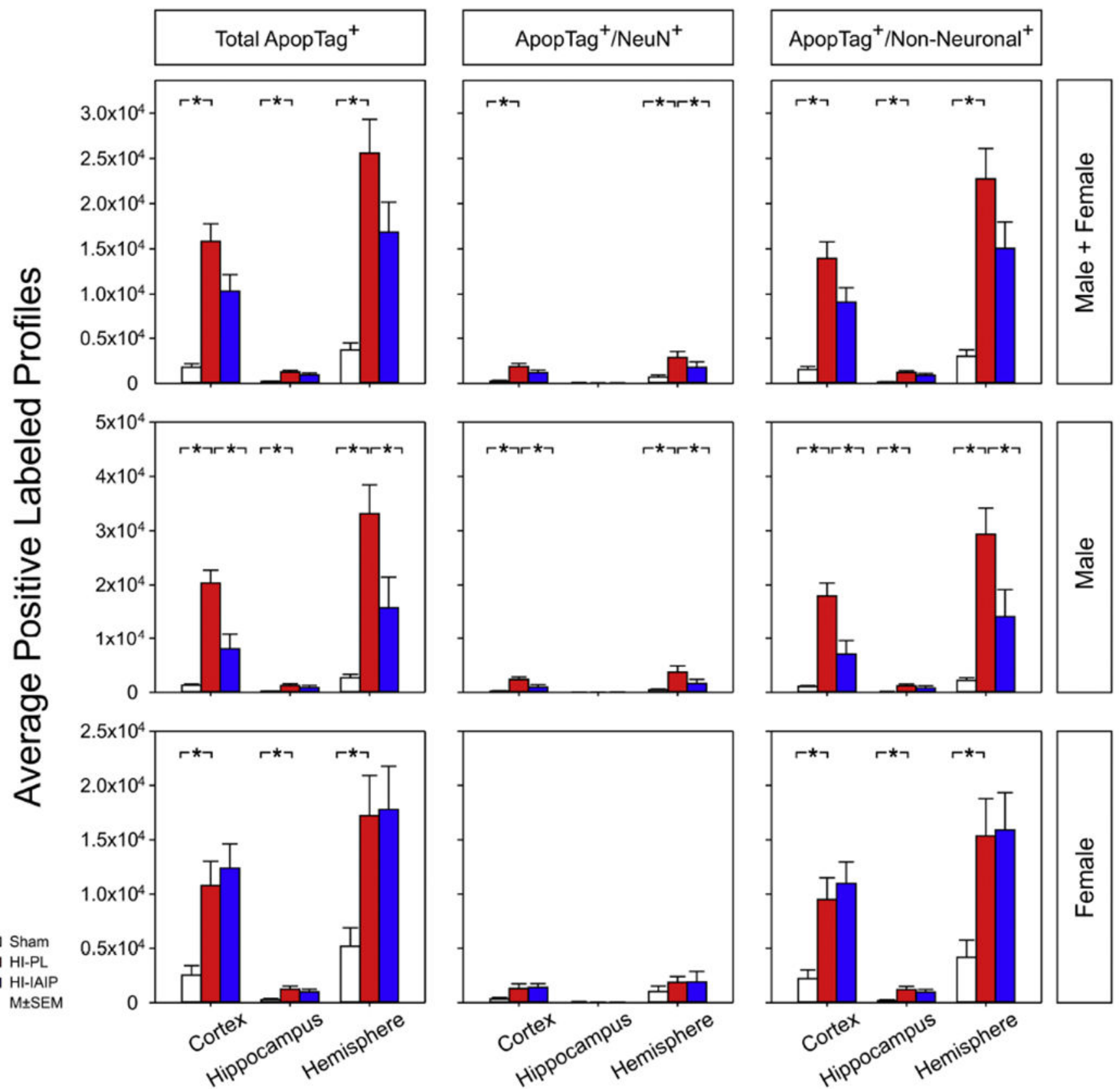

Fig. 8.

The average positive-labeled profiles of total ApopTag ${ }^{+}, \mathrm{ApopTag}^{+} / \mathrm{NeuN}^{+}, \mathrm{ApopTag}^{+} / \mathrm{Non}-$
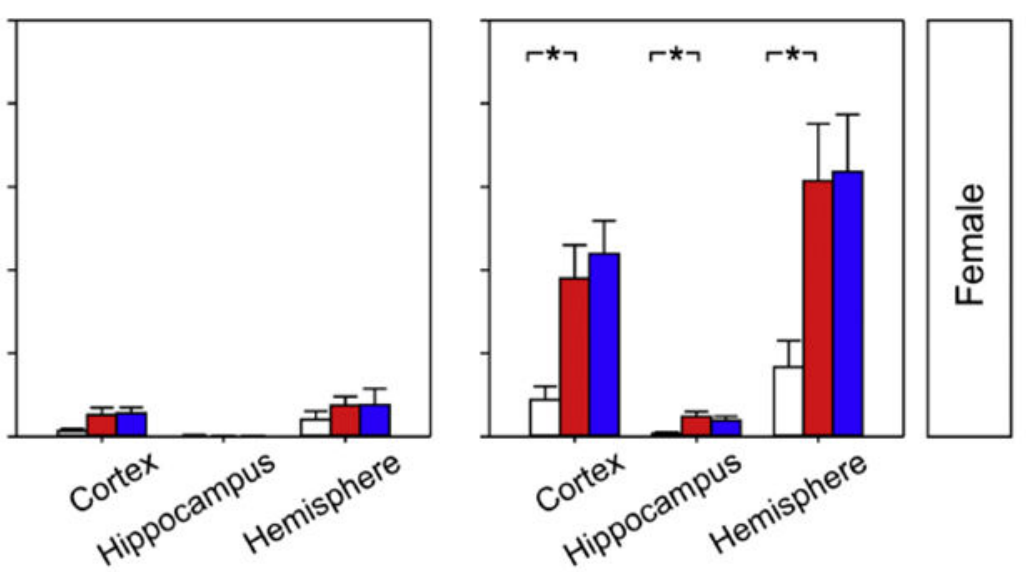

Neuronal ${ }^{+}$profiles for the cerebral cortex, hippocampus and total hemisphere in the Sham, HI-PL and HI-IAIP groups. Top panel reports data for males + females, middle panel for males, and bottom panel for females. Values are mean \pm SEM. Sham: male $n=9-12$, female $\mathrm{n}=4-8$; HI-PL: male $\mathrm{n}=7-10$, female $\mathrm{n}=7-9$; HI-IAIP: male $\mathrm{n}=6-9$, female $\mathrm{n}=5-10$. $* P<0.05$. 

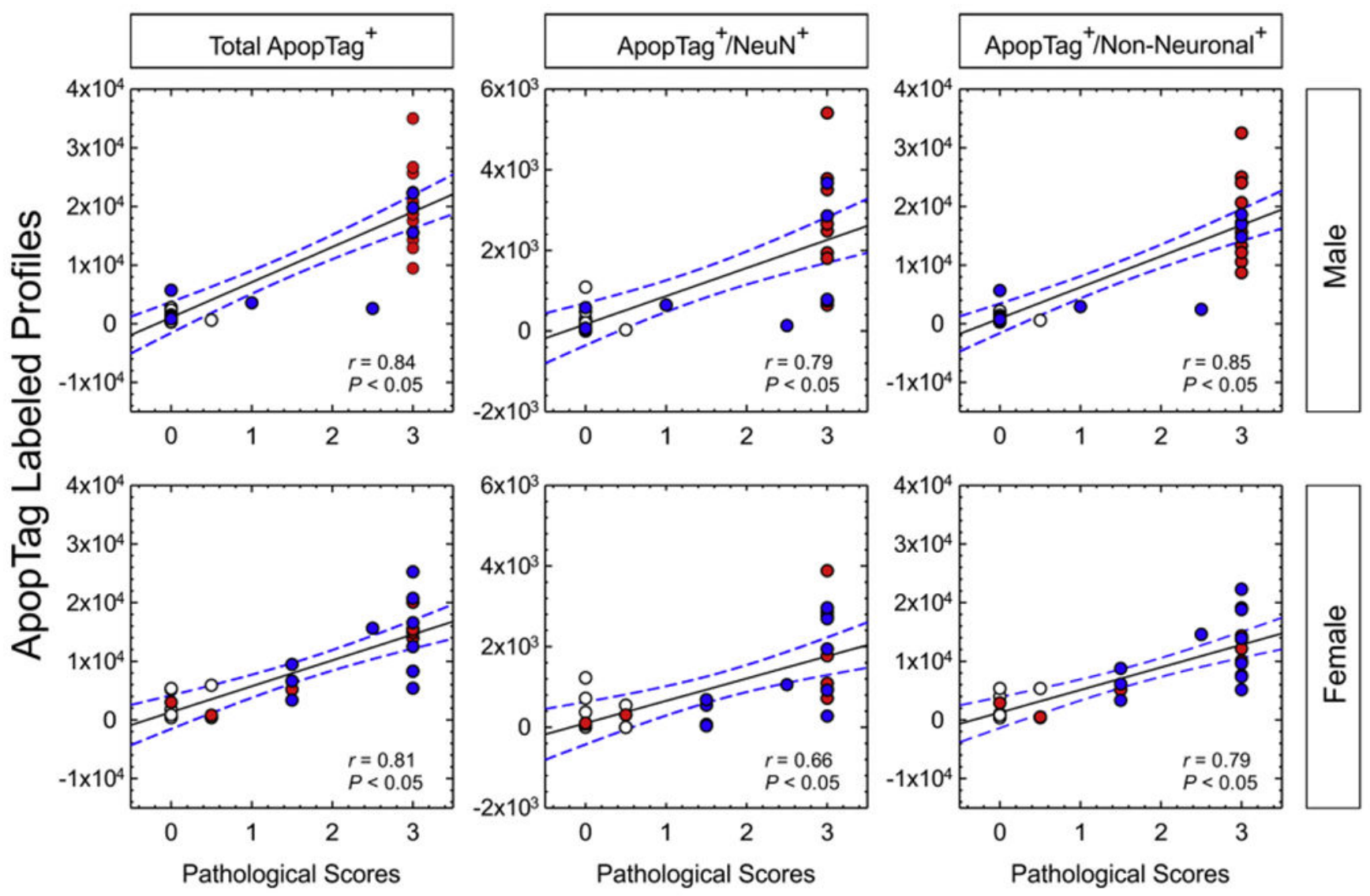

O Sham, O HI-PL,

HI-IAIP,

regression line,

$95 \%$ confidence band

Fig. 9.

The average positive-labeled profiles of total ApopTag ${ }^{+}, \mathrm{ApopTag}^{+} / \mathrm{NeuN}^{+}, \mathrm{ApopTag}^{+} / \mathrm{Non}-$ $\mathrm{Neuronal}^{+}$profiles plotted on the $\mathrm{y}$-axis against the pathological scores for the parietal cortex on the $\mathrm{x}$-axis for the males (top panel) and females (bottom panel). Spearman rank test, $* P<0.05$. Solid line is the regression line. Dashed lines are the $95 \%$ confidence intervals. 

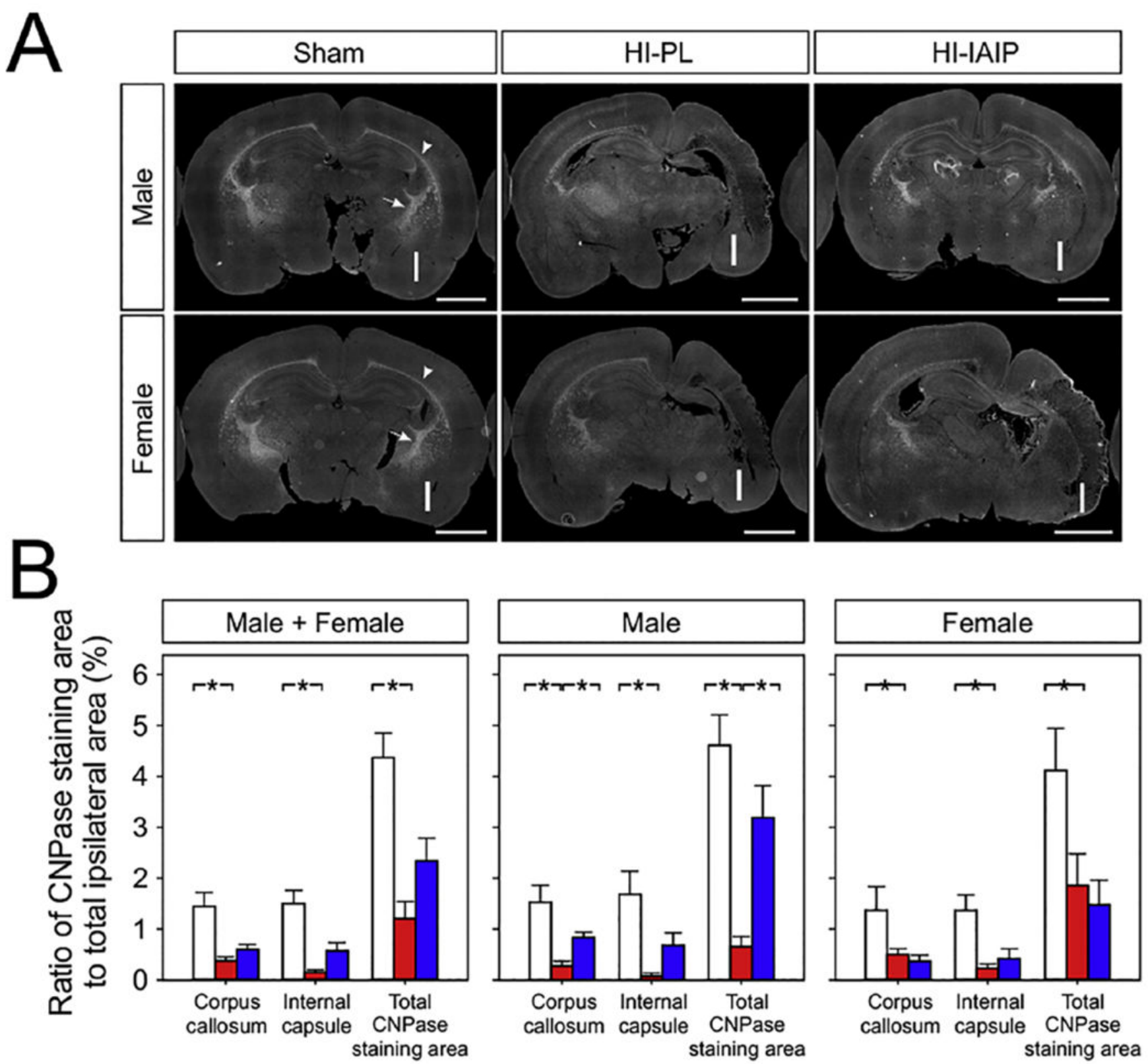

Fig. 10.

(A) Representative immunohistochemical stitched, 8-bit grayscale images containing CNPase-labeled cells of the Sham, HI-PL and HI-IAIP male (top row) and female (bottom row) neonatal rats. The "I" designates the side of the brain ipsilateral to the carotid artery ligation. The white, dashed arrows indicate the CNPase staining area in the internal capsule. The white arrowheads indicate the CNPase staining area in the corpus callosum. Scale bar = $2 \mathrm{~mm}$. (B) The average percent ratio of CNPase-positive area to the total area ipsilateral to HI for the corpus callosum, internal capsule, and total hemisphere in the Sham, HI-PL and HI-IAIP groups for males + females, males, and females. Values are mean \pm SEM. Sham: male $\mathrm{n}=5$, female $\mathrm{n}=5$; HI-PL: male $\mathrm{n}=6$, female $\mathrm{n}=5$; HI-IAIP: male $\mathrm{n}=7$, female $\mathrm{n}=$ 7. $* P<0.05$. 

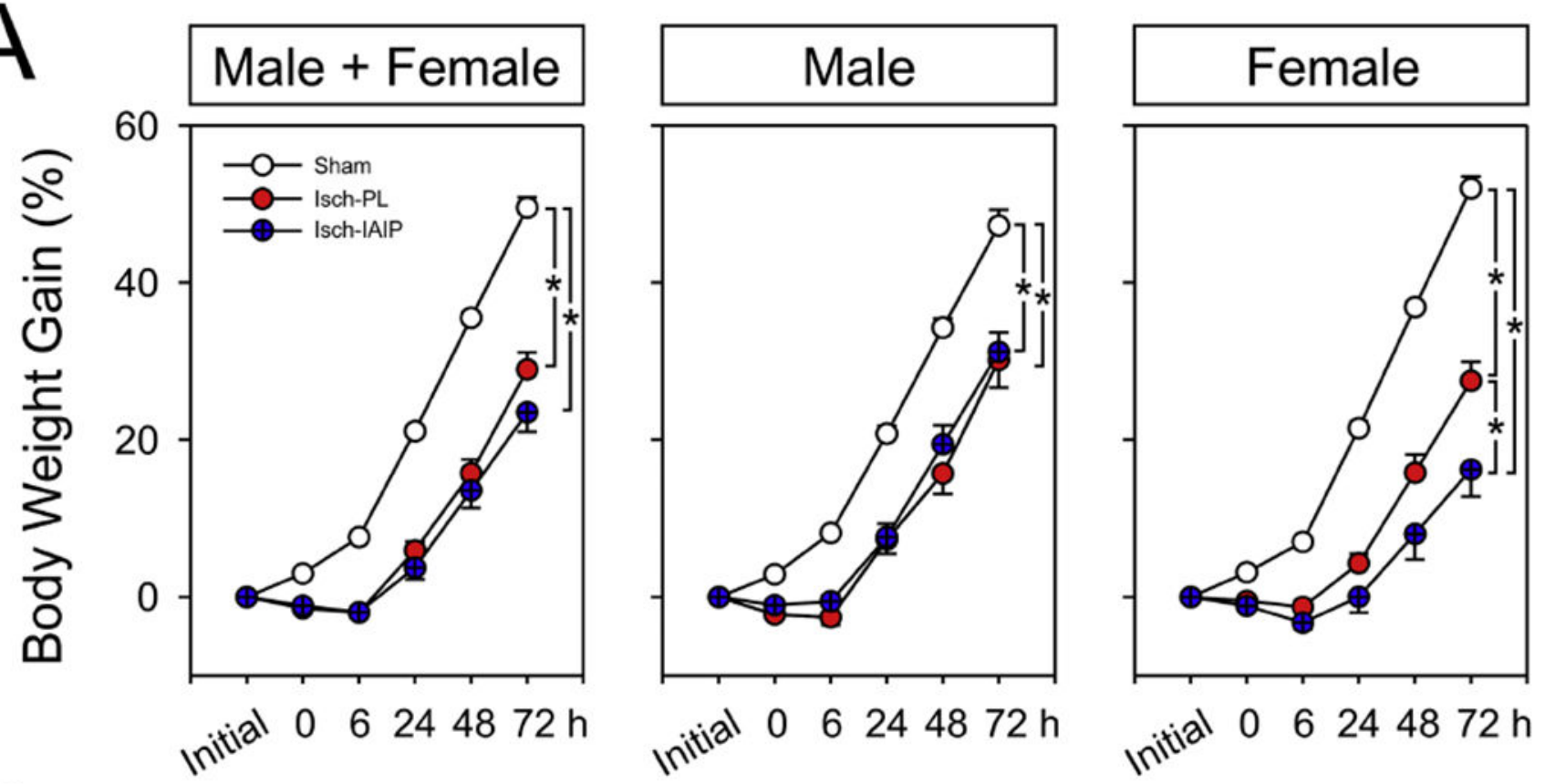

B

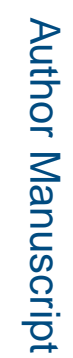
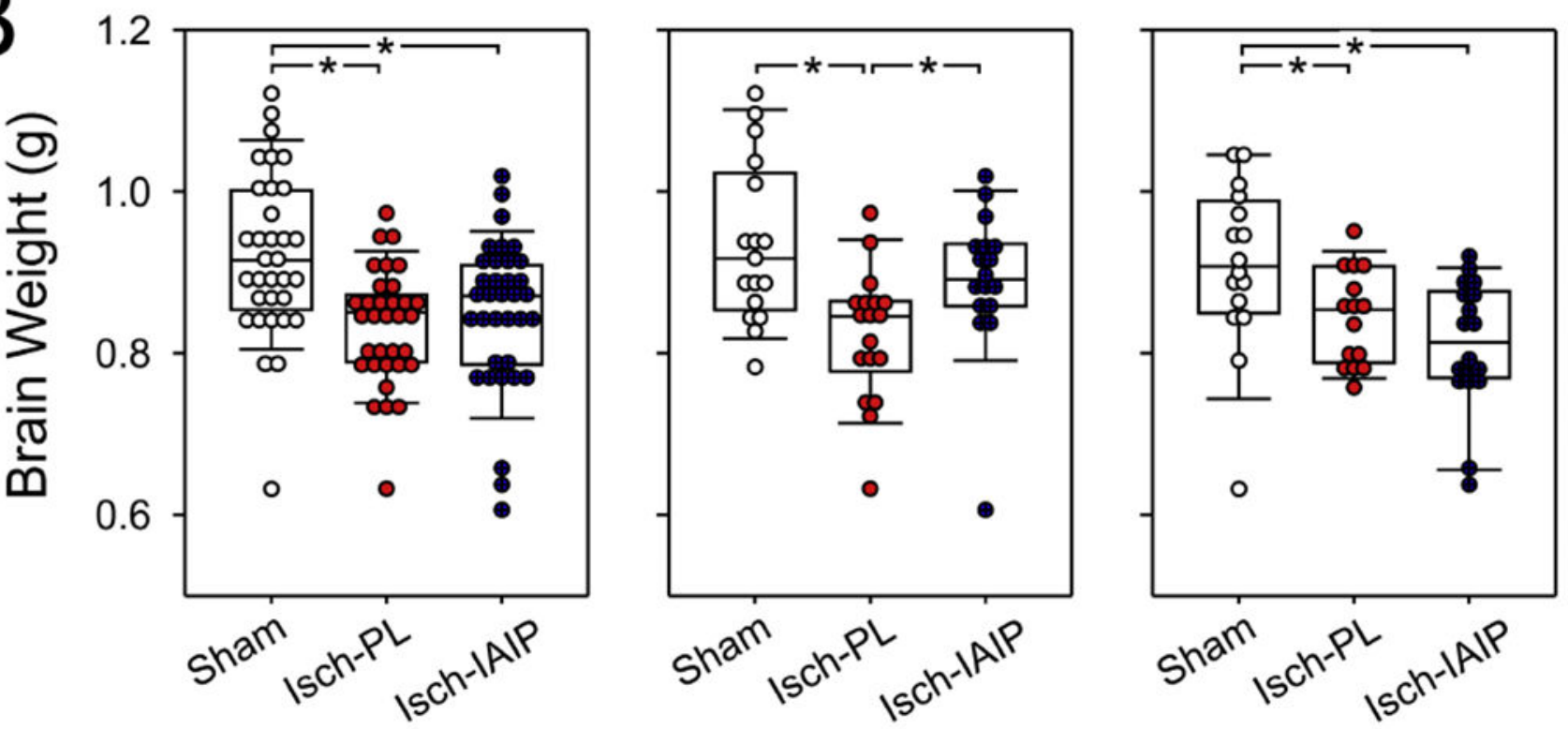

Fig. 11.

Body weight gain (\%) and brain weights (g) in the Sham, HI-PL and HI-IAIP groups after delayed treatment with IAIPs or placebo in neonatal rats exposed to HI brain injury. (A) Percent body weight gain plotted on the $y$-axis against study time in hours on the $\mathrm{x}$-axis for the males + females, males and females. Sham: male $n=17$, female $n=16$; HI-PL: male $n=$ 18 , female $\mathrm{n}=15$; HI-IAIP: male $\mathrm{n}=17$, female $\mathrm{n}=18$. Values are mean \pm SEM. (B) Brain weights in the males + females, males, and females plotted as dot box plots showing the median, and $5^{\text {th }}$ and $95^{\text {th }}$ percentiles. Sham: male $\mathrm{n}=17$, female $\mathrm{n}=16$; HI-PL: male $\mathrm{n}=18$, female $\mathrm{n}=15$; HI-IAIP: male $\mathrm{n}=17$, female $\mathrm{n}=18 . * P<0.05$. 

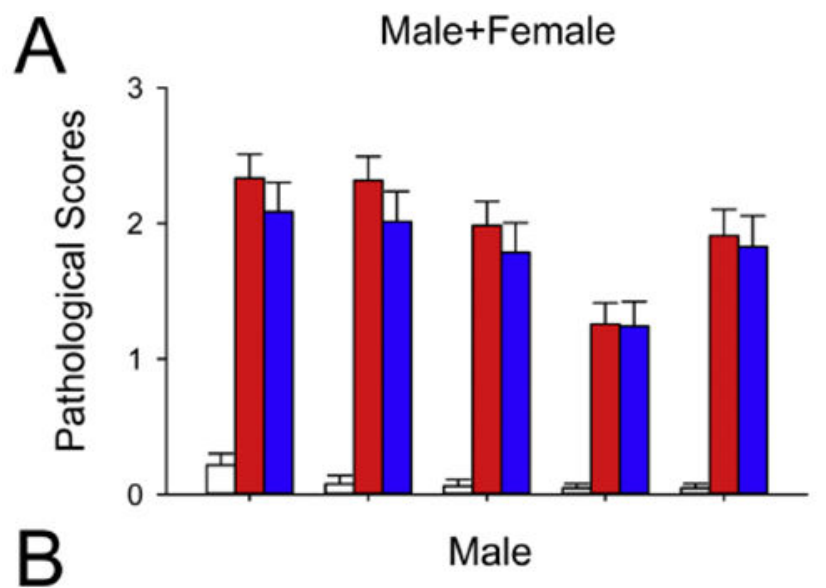

Male
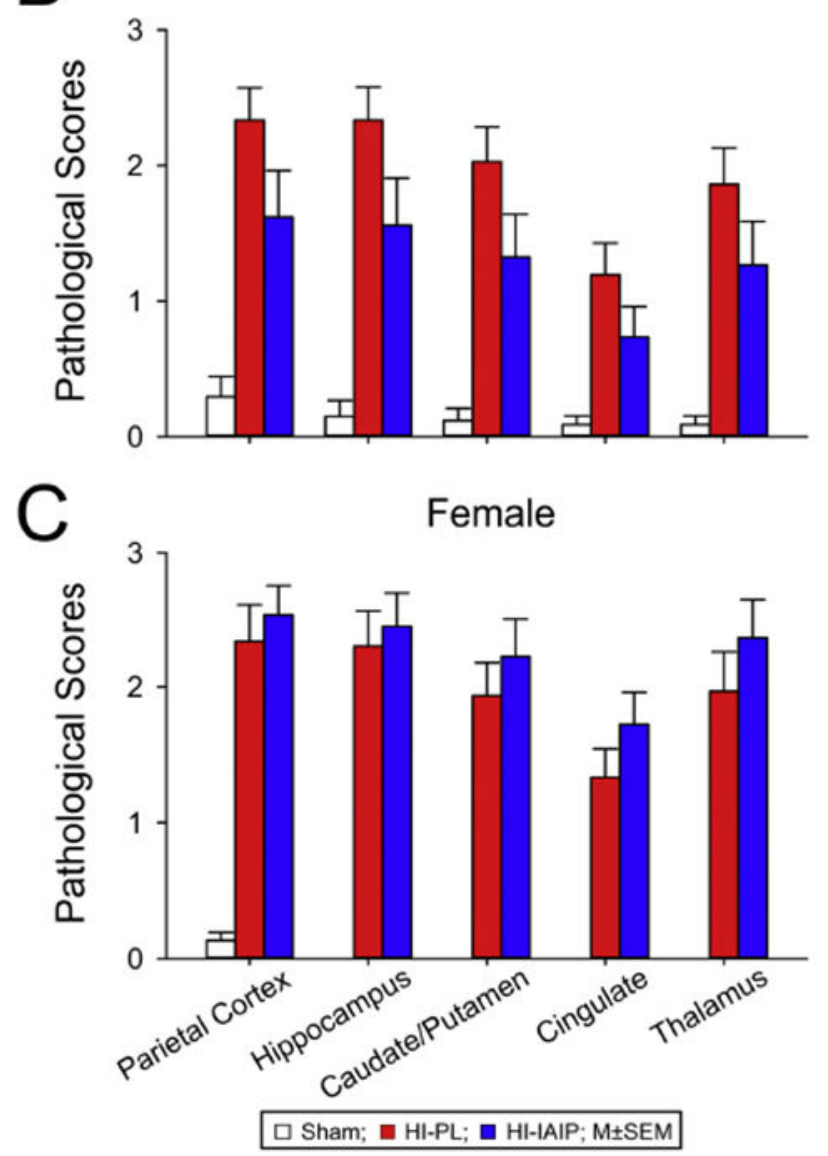

Fig. 12.

Pathological scores plotted on the y-axis for the parietal cortex, hippocampus, caudate/ putamen, cingulate and thalamus on the $\mathrm{x}$-axis for the $(\mathrm{A})$ male + females, $(\mathrm{B})$ males and $(\mathrm{C})$ females in the Sham, HI-PL, and HI-IAIP groups after delayed treatment with placebo or IAIPs. Values are mean \pm SEM. Sham: male $n=17$, female $n=15$; HI-PL: male $n=18$, female $n=15$; HI-IAIP: male $n=17$, female $n=18$. 